\title{
Electron and Phonon Properties of Graphene: Their Relationship with Carbon Nanotubes
}

\author{
J.-C. Charlier ${ }^{1}$, P. C. Eklund ${ }^{2}$ J. Zhu ${ }^{2}$, A. C. Ferrari ${ }^{3}$ \\ 1 Unité de Physico-Chimie et de Physique des Matériaux \\ Université Catholique de Louvain \\ 1 Place Croix du Sud, 1348 Louvain-la-Neuve, Belgium \\ jean-christophe.charlier@uclouvain.be \\ 2 Department of Physics, The Pennsylvania State University \\ 104 Davey Lab, University Park, PA 16802-6300, USA \\ pce3@psu.edu, jzhu@phys.psu.edu \\ 3 Engineering Department, Cambridge University \\ 9 JJ Thomson Avenue, Cambridge CB3 OFA, UK \\ acf26@eng. cam.ac.uk
}

\begin{abstract}
The discovery of Novoselov et al. (2004) of a simple method to transfer a single atomic layer of carbon from the c-face of graphite to a substrate suitable for the measurement of its electrical and optical properties has led to a renewed interest in what was considered to be before that time a prototypical, yet theoretical, two-dimensional system. Indeed, recent theoretical studies of graphene reveal that the linear electronic band dispersion near the Brillouin zone corners gives rise to electrons and holes that propagate as if they were massless fermions and anomalous quantum transport was experimentally observed. Recent calculations and experimental determination of the optical phonons of graphene reveal Kohn anomalies at high-symmetry points in the Brillouin zone. They also show that the BornOppenheimer principle breaks down for doped graphene. Since a carbon nanotube can be viewed as a rolled-up sheet of graphene, these recent theoretical and experimental results on graphene should be important to researchers working on carbon nanotubes. The goal of this contribution is to review the exciting news about the electronic and phonon states of graphene and to suggest how these discoveries help understand the properties of carbon nanotubes.
\end{abstract}

\section{Introduction}

The fundamental building block of both a graphite crystal and a carbon nanotube is the graphene layer shown schematically in Fig. 1a. Such an atomic structure is characterized by two types of $\mathrm{C}-\mathrm{C}$ bonds $(\sigma, \pi)$ constructed from the four valence orbitals $\left(2 \mathrm{~s}, 2 \mathrm{p}_{x}, 2 \mathrm{p}_{y}, 2 \mathrm{p}_{z}\right)$, where the $z$-direction is perpendicular to the sheet. Three $\sigma$-bonds join a $\mathrm{C}$ atom to its three neighbors. They are quite strong, leading to optical-phonon frequencies much higher than observed in diamond. In addition, the $\mathrm{C}-\mathrm{C}$ bonding is enhanced by a fourth bond associated with the overlap of $p_{z}$ (or $\pi$ ) orbitals. Graphite is comprised of coherently stacked layers of graphene. Two layers ( $A$ and $B$ ) are needed to define the unit cell in graphite. The three-dimensional (3D)

\footnotetext{
A. Jorio, G. Dresselhaus, M. S. Dresselhaus (Eds.): Carbon Nanotubes,

Topics Appl. Physics 111, 673-709 (2008)

Springer-Verlag Berlin Heidelberg 2008
} 
structure of graphite is therefore held together by a weak interlayer van der Waals (vdW) force. A single-wall carbon nanotube (SWNT), on the other hand, is a seamless cylinder of graphene. SWNTs are analogously bound in a bundle by the vdW force. The electronic properties of graphene, graphite and carbon nanotubes are determined by the bonding $\pi$ - and antibonding $\pi^{*}$ orbitals that form wide electronic valence and conduction bands (Fig. 1b). As the tube diameter decreases, the bond angle between the $\sigma$ and $\pi$ orbitals increases slightly, introducing curvature effects into the electronic and phonon properties of SWNTs.

Theoretical calculations show that the $\pi$-band overlap in graphite disappears as the layers are further separated over their equilibrium distance in graphite. This leads to decoupled graphene layers that can be described as a zero-gap semiconductor. The $\pi$-band electronic dispersion for graphene near the six corners of the 2D hexagonal Brillouin zone is found to be linear. Thus, "cones" of carriers (holes and electrons) appear in the corners of a 2D Brillouin zone whose points touch at the Fermi energy, as shown in Fig. 1c. The linear electronic band dispersion leads to the term "massless Dirac fermions" for these carriers. The six points where the cones touch are referred to as the "Dirac" points in the graphene literature.

Graphene, because of its structural simplicity (two atoms per unit cell), has been extensively investigated in theory for the past 60 years [1]. However, only recently has it been possible to produce ultrathin films containing a countable number $(n)$ of graphene layers (i.e., $1<n<20$ ). The synthetic techniques fall into two categories. The first approach exploits the weak bonding between the graphene layers by pealing off an $n$-graphene layer ( $n \mathrm{GL}$ ) by chemical means [2-6] or by mechanical means [7, 8]. Mechanical separation of $n$ GLs from the parent crystal involves rubbing the freshly cleaved quasi$2 \mathrm{D}$ system against a smooth substrate. This technique has allowed graphene and $n$ GLs to be studied in the laboratory for the first time [7]. It is simple, inexpensive and produces graphene and $n$ GL flakes of very high quality. However, this approach lacks the scalability required by mass device production. The second approach grows epitaxial graphitic films by thermal decomposition of $\mathrm{SiC}$ [9-12]. This technique has the potential of producing large-area lithography-compatible films and is rapidly advancing at the moment.

In this contribution, we review the connection between the electron and phonon states in graphene $(n$ GLs) and SWNTs. We begin with a discussion of the electronic states. Recent theoretical arguments and experiments are converging on a picture of free carriers in graphene near the Fermi energy behaving as massless Dirac fermions propagating at $\sim 1 / 100$ the speed of light, i.e., they do not behave as simple electrons or holes with an effective mass. Experimental observations of an anomalous integer quantum Hall effect in graphene has been reported recently and has created a lot of attention $[13,14]$. These and other interesting experimental electronic transport results are reviewed as well [15] with further discussion provided in the contributions in this volume by Ando and by Spataru et al. We then discuss 


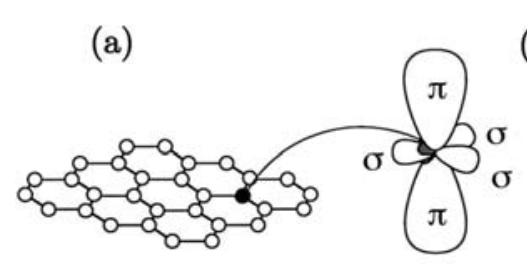

(b)

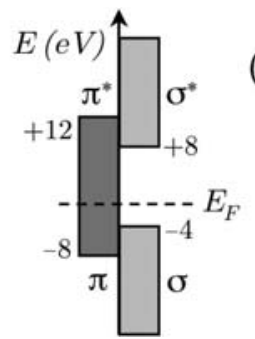

(c)

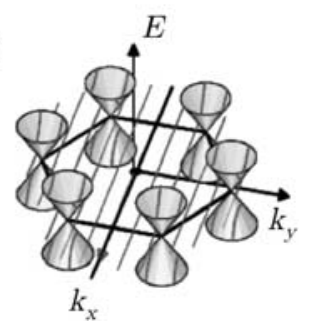

Fig. 1. Illustration of the carbon valence orbitals. (a) The three inplane $\sigma$ (s, $\left.p_{x}, p_{y}\right)$ orbitals in graphene and the $\pi\left(p_{z}\right)$ orbital perpendicular to the sheet. The inplane $\sigma$ and the $\pi$ bonds in the carbon hexagonal network strongly connect the carbon atoms and are responsible for the large binding energy and the elastic properties of the graphene sheet. The $\pi$ orbitals are perpendicular to the surface of the sheet. The corresponding bonding and the antibonding $\sigma$ bands are separated by a large energy gap of $\sim 12 \mathrm{eV}(\mathbf{b})$, while the bonding and antibonding $\pi$ states lie in the vicinity of the Fermi level $\left(E_{\mathrm{F}}\right)$. Consequently, the $\sigma$ bonds are frequently neglected for the prediction of the electronic properties of graphene around the Fermi energy. Dirac cones located at the six corners of the 2D Brillouin zone are illustrated in (c)

the optical phonons in graphene that are directly accessible by Raman spectroscopy and give rise to the most prominent Raman peaks when graphene is folded into nanotubes. We focus on their coupling to electrons, which is key to understanding many phenomena in graphene and nanotubes.

For example, it was recently argued that in doped graphene, the adiabatic Born-Oppenheimer approximation [16], valid in many solid-state systems, breaks down [17]. The electron-phonon interaction in graphene has also been carefully re-examined and has been recently shown to give rise to Kohn anomalies in the phonon dispersion at important points $(\Gamma, K)$ in the Brillouin zone where the phonons can be studied by Raman spectroscopy [1821]. Many of these new ideas proposed for graphene and $n$ GLs carry over to nanotubes. Kohn anomalies are responsible for the different Raman spectra of metallic and semiconducting nanotubes, and non-Born-Oppenheimer effects strongly shape the Raman spectra of doped and annealed nanotubes [19, 22]. These connections are also discussed in this review.

\section{Electronic Properties and Transport Measurements}

\subsection{Graphene}

\subsubsection{Electronic Band Structure}

Figure 2 shows the ab-initio calculations of the electronic bands of graphene along the high-symmetry $M-\Gamma-K$ directions [23]. Its space group ( $P 3 m)$ 
contains a mirror-plane symmetry, allowing symmetric $\sigma$ and antisymmetric $\pi$ states to be distinguishable. In a $2 \mathrm{D}$ crystal, a parallel mirror symmetry operation separates the eigenstates for the whole Brillouin zone, and not only along some high-symmetry axis. The $\pi$ and $\pi^{*}$ bands touch at the corners of the hexagonal Brillouin zone. Such corners are labeled by their momentum vector usually denoted by $K$ and $K^{\prime}$. Consequently, graphene is a special semimetal or zero-gap semiconductor, whose intrinsic Fermi surface is reduced to the six points at the corners of the $2 \mathrm{D}$ hexagonal Brillouin zone. Close to the Fermi energy, the $\pi$ and $\pi^{*}$ bands are quasilinear (linear very close to $K, K^{\prime}$ ) (Fig. 1c), in contrast with the usual quadratic energy-momentum relation obeyed by electrons at band edges in conventional semiconductors. When several interacting graphene planes are stacked, as in $n$ GLs or in a perfect graphite crystal, the former antisymmetric $\pi$ bands are split (owing to bonding or antibonding patterns), whereas the $\sigma$ bands are much less affected by the stacking.

The graphene sheet is thus highly specific for this linear energy-momentum relation. The electronic group velocity, estimated at the Dirac points, is quite high: $\sim 1 \times 10^{6} \mathrm{~m} / \mathrm{s}$. Consequently, graphene exhibits electronic properties for a $2 \mathrm{D}$ gas of charged particles described by an equation of the form of the relativistic Dirac equation rather than the nonrelativistic Schrödinger equation with an effective mass. Indeed, in graphene, charge carriers mimic particles with zero mass and an effective "speed of light" $c^{*} \sim 1 \times 10^{6} \mathrm{~m} / \mathrm{s}$. Recently, graphene has revealed a variety of unusual transport phenomena characteristic of two-dimensional Dirac fermions, such as an anomalous integer quantum Hall effect, a "minimum" conductivity of order $4 e^{2} / h$ even when the carrier concentration tends to zero, a cyclotron mass $m_{\mathrm{c}}$ of massless carriers with an energy $E$ described by $E=m_{\mathrm{c}} c^{* 2}$, and Shubnikov-de Haas oscillations that exhibit a phase shift of $\pi$ due to Berry's phase $[13,14]$. These remarkable properties will be described in the next section.

The electronic structure of graphene can be reasonably well described using a rather simple tight-binding Hamiltonian, leading to analytical solutions for their energy dispersion and related eigenstates [23]. Since the bonding and antibonding $\sigma$ bands are well separated in energy $(>10 \mathrm{eV}$ at $\Gamma$ ), they are frequently neglected in semiempirical calculations since they are too far away from the Fermi level to play a role. Only the remaining two $\pi$ bands are thus needed to describe the electronic properties of graphene.

When the atoms are placed onto the graphene hexagonal lattice (Fig. 1a), the electronic wavefunctions from different atoms overlap. However, such an overlap between the $\mathrm{p}_{z}(\pi)$ orbitals and the $s$ or the $p_{x}$ and $p_{y}$ orbitals is strictly zero by symmetry. Consequently, the $\mathrm{p}_{z}$ electrons, which form the $\pi$ bands in graphene, can be treated independently from the other valence electrons. Within this $\pi$-band approximation, it is easy to describe the electronic 

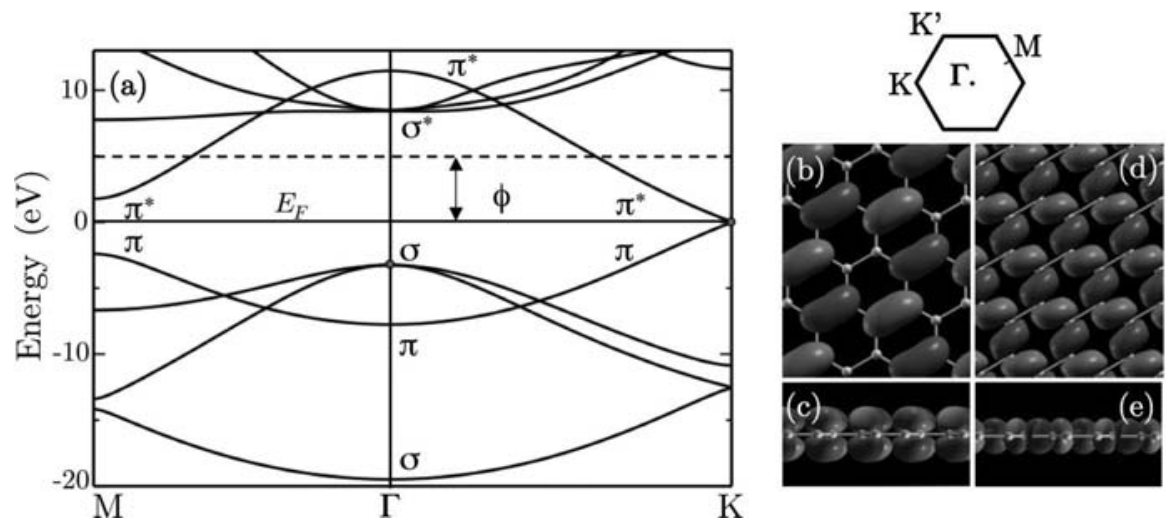

Fig. 2. (a) Electronic band structure of graphene from ab-initio calculations [23]. The bonding $\sigma$ and the antibonding $\sigma^{*}$ bands are separated by a large energy gap. The bonding $\pi$ (highest valence band) and the antibonding $\pi^{*}$ (lowest conduction band) bands touch at the $K\left(K^{\prime}\right)$ points of the Brillouin zone. The Fermi energy $\left(E_{\mathrm{F}}\right)$ is set to zero and $\phi$ indicates the work function (by the dashed horizontal line). Above the vacuum level $\phi$, the states of the continuum are difficult to describe and merge with the $\sigma^{*}$ bands. The $2 \mathrm{D}$ hexagonal Brillouin zone is illustrated with the high-symmetry points $\Gamma, M, K$ and $K^{\prime}$. (b,c) $\pi$ state at $K$ and (d,e) $\sigma$-state at $\Gamma$ seen, respectively, from above and from the side of the graphene plane. Note that the $\pi$ wavefunction cancels on a hexagonal sublattice due to the $\left.e^{(i K} r\right)$ phase factor. The $\pi(\sigma)$ state is odd (even) with respect to the graphene plane reflection. Courtesy of Zanolli, 2007, unpublished

spectrum of the total Hamiltonian and to obtain the dispersion relations restricted to first-nearest-neighbor interactions only:

$$
E^{ \pm}\left(k_{x}, k_{y}\right)= \pm \gamma_{0} \sqrt{1+4 \cos \frac{\sqrt{3} k_{x} a}{2} \cos \frac{k_{y} a}{2}+4 \cos ^{2} \frac{k_{y} a}{2}}
$$

where $a=\sqrt{3} a_{\mathrm{C}-\mathrm{C}}\left(a_{\mathrm{C}-\mathrm{C}}=1.42 \AA\right.$ is the carbon-carbon distance in graphene) and $\gamma_{0}$ is the transfer integral between first-neighbors $\pi$ orbitals (typical values for $\gamma_{0}$ are $\left.2.9-3.1 \mathrm{eV}\right)$. The $\boldsymbol{k}=\left(k_{x}, k_{y}\right)$ vectors that belong to the first hexagonal Brillouin zone (BZ) constitute the ensemble of available electronic momenta.

With one $p_{z}$ electron per atom in the $\pi-\pi^{*}$ model (the three other $s, p_{x}$, $p_{y}$ electrons fill the low-lying $\sigma$ band), the $(-)$ band (negative energy branch) in (1) is fully occupied, while the $(+)$ branch is totally empty. These occupied and unoccupied bands touch at the $K$ and $K^{\prime}$ points. The Fermi level $E_{\mathrm{F}}$ (or charge-neutrality point) is chosen as the zero-energy reference in Fig. 2 and the Fermi surface is defined by the set of $K$ and $K^{\prime}$ points. Since the occupied and unoccupied bands touch at the zone corners, the graphene sheet displays a semimetallic, or zero-gap semiconducting character. 


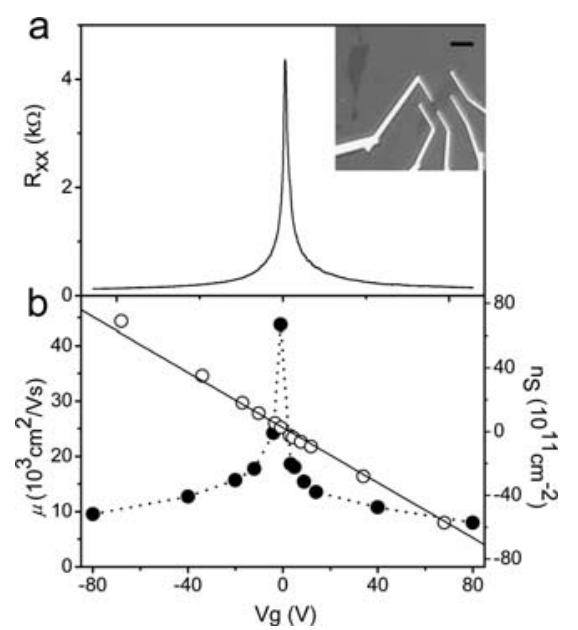

Fig. 3. Resistivity, mobility and carrier density as a function of gate voltage $V_{\mathrm{g}}$ in a single-layer graphene fieldeffect transistor device. (a) $V_{\mathrm{g}}$-dependent $R_{x x}$ showing a finite value at the Dirac point. The resistivity $\rho_{x x}$ can be calculated from $R_{x x}$ using the geometry of the device. The inset is an image of a graphene device on a $\mathrm{Si}: \mathrm{SiO}_{2}$ substrate. The $\mathrm{Si}$ is the bottom gate; five top electrodes formed via e-beam lithography are shown in the inset. Scale bar $5 \mu \mathrm{m}$. (b) Mobility $\mu$ and carrier density $n_{s}$ as a function of $V_{\mathrm{g}}$. The mobility (dotted curve) diverges artificially at the Dirac point due to a finite resistivity. Adapted from [14]

Expanding (1) at $K\left(K^{\prime}\right)$ yields the linear $\pi$ and $\pi^{*}$ bands for Dirac fermions:

$$
E^{ \pm}(\boldsymbol{\kappa})= \pm \hbar v_{\mathrm{F}}|\boldsymbol{\kappa}|
$$

where $\boldsymbol{\kappa}=\boldsymbol{k}-K$, and $v_{\mathrm{F}}$ is the electronic group velocity given by:

$$
v_{\mathrm{F}}=\sqrt{3} \gamma_{0} a / 2 \hbar \text {. }
$$

In graphene, $v_{\mathrm{F}} \approx 1 \times 10^{6} \mathrm{~m} / \mathrm{s}$. The resulting cone structure at the six Brillouin zone corners is shown in Fig. 1c.

The linear dispersion given by (2) is the solution to the following effective Hamiltonian at the $K\left(K^{\prime}\right)$ point [24]:

$$
\boldsymbol{H}=\hbar v_{\mathrm{F}}(\boldsymbol{\sigma} \boldsymbol{\kappa})
$$

where $\boldsymbol{\kappa}=-i \nabla$, and $\boldsymbol{\sigma}$ 's are the pseudospin Pauli matrices operating in the space of the electron amplitude on the A-B sublattices of graphene.

In the next section, we briefly describe some of the unusual properties of graphene [15] derived from the linear dispersion and the "chiral" nature of the quasiparticles defined by (2) and (4).

\subsubsection{Transport Measurements in Single-Layer Graphene}

Figure 3 shows the resistivity $\rho_{x x}$, density $n_{s}$ and mobility $\mu$ of $2 \mathrm{D}$ carriers in a single-layer graphene field-effect transistor (FET) as a function of gate voltage [14]. Graphene FETs are fabricated with standard lithography and etching techniques using mechanically cleaved graphene flakes [25]. A degenerately doped silicon substrate with a thin oxide overlayer serves as an 
insulated gate electrode that can continuously tune $E_{\mathrm{F}}$, and therefore the 2D carrier density $n_{S}$ across the charge-neutrality point, covers a range of roughly $\pm 1 \times 10^{13} / \mathrm{cm}^{2}$. The conduction is symmetric about the Dirac point as shown in Fig. 3a, indicating that the electron and hole mobilities are approximately equal. In the high carrier density regime, the mobility is found to be roughly carrier-density independent and temperature independent. Reported mobility values span a wide range $2000<\mu<20000 \mathrm{~cm}^{2} /(\mathrm{Vs})$, with the high end representing a long mean-free path of $\sim 0.3 \mu \mathrm{m}$, comparable to that of SWNTs. The weak temperature dependence found for $\mu$ indicates that impurity or defect scattering is the dominant scattering mechanism and provides room for future device improvement $[13,26]$. Possible sources for scattering centers include adsorbents, defects in the graphene lattice and ionized impurities in the $\mathrm{SiO}_{2}$ substrate. The carrier-density dependence of the conductivity (not shown) near the Dirac point is found to sensitively depend upon the range of the scatterers [27] and varies from device to device.

Figure 3 also reveals a remarkable property of 2D carriers in graphene. Although the carrier density approaches zero at the Dirac point, the $2 \mathrm{D}$ resistivity $\rho_{x x}$ or conductivity $\sigma_{x x}$ remains finite. Such behavior, dubbed the "minimum" conductivity, has been observed experimentally in many graphene devices with $\rho_{x x}$ ranging from 2 to $7 \mathrm{k} \Omega$ at low temperature [13,14]. A slightly larger value of $\rho_{x x}$ from 6 to $9 \mathrm{k} \Omega$ is also reported for bilayer graphene [28]. The origin of this finite conductivity $\sim 4 e^{2} / h$ remains unclear at the moment. In a real device, ionized impurities in the $\mathrm{SiO}_{2}$ substrate or the rippling of the graphene sheet [29] may lead to local puddles of electrons and holes with finite densities and result in finite conduction through the device [30]. On the other hand, calculations show that a minimum quantum conductivity (often $\left.4 e^{2} / h \pi\right)$ can also arise intrinsically from the linear excitation spectrum of Dirac fermions and/or the chiral nature of the quasiparticles (for a more detailed review, see [15]). However, present experiments seem to converge at $4 e^{2} / h$. More experiments, particularly with cleaner substrates or suspended devices, are needed to clarify this intriguing issue.

In a magnetic field perpendicular to the graphene plane, the linear excitation spectrum of Dirac fermions evolves into discrete Landau levels (LLs) whose energies are given $[31,32]$ in terms of the LL index by:

$$
E_{n}= \pm v_{\mathrm{F}} \sqrt{2 e \hbar|n| B}
$$

The $\sqrt{B}$ dependence of $E_{n}$, which distinguishes graphene from conventional $2 \mathrm{D}$ semiconductors (that have LLs linear in $B$ ), has been confirmed by recent spectroscopic measurements [33-35]. The large splitting in graphene $\left(\Delta E_{0,1}=240 \mathrm{meV}\right.$ at $\left.45 \mathrm{~T}\right)$ leads to the observation of the quantum Hall effect at room temperature [36].

Including spin and sublattice degeneracy, the number of states for each LL is $4 e B / h$. In graphene, because of the touching bands at the $K\left(K^{\prime}\right)$ points, the $n=0 \mathrm{LL}$ is shared equally between electrons and holes. This counting scheme 

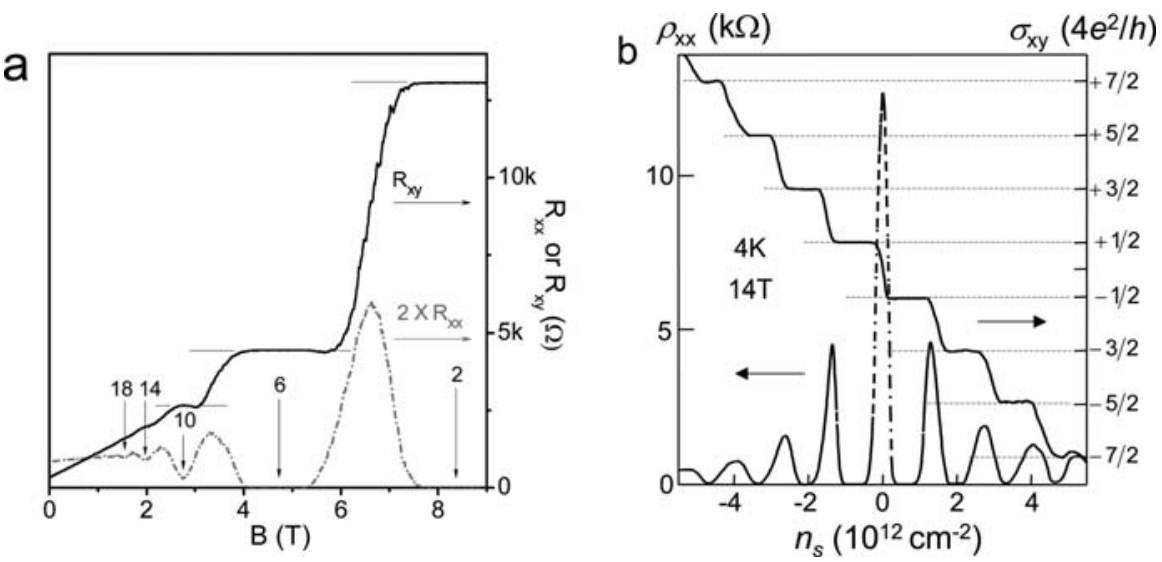

Fig. 4. Anomalous-integer quantum Hall effect in single-layer graphene. (a) Magnetic-field sweeps of Hall resistance $R_{x y}$ (solid) and longitudinal resistance $R_{x x}$ (dash-dotted) at fixed carrier density (or $V_{\mathrm{g}}$ ) showing plateaus in $R_{x y}$ quantized at $h / e^{2} \nu$ and the corresponding zero-resistance minima in $R_{x x}$. Arrows indicate filling factor $\nu=n_{s} h / e B=4(n+1 / 2)$, where $n_{s}$ is the carrier density and $n$ the LL index. Adapted from [14]. (b) Hall conductivity $\sigma_{x y}$ (solid) and longitudinal resistivity $\rho_{x x}$ (dashed) as a function of carrier density at $B=14 \mathrm{~T}$. Here, $\sigma_{x y}$ is calculated as $\sigma_{x y}=\rho_{x y} /\left(\rho_{x x}^{2}+\rho_{x y}^{2}\right)$ and is observed to be quantized as $\sigma_{x y}=4(n+1 / 2) e^{2} / h$. Adapted from [13]

gives rise to the occurrence of the quantum Hall effect at half-integer LL indices (Fig. 4), which can also be viewed as the result of a conventional integer sequence with a phase shift of $\pi$ known as Berry's phase [37]. This $\pi$ phase shift arises from the linear dispersion of the Dirac fermions and serves as a sensitive indicator of the existence of such an excitation spectrum. It persists to lower magnetic fields, where quantum Hall states turn into magnetoresistance (Shubnikov-de Haas or SdH) oscillations [38]. The magnitude of the phase shift can be accurately extracted from the LL fan diagrams (Fig. 5a) [13, 14].

Although the light-like linear electronic band dispersion implies massless particles, Dirac fermions moving in a magnetic field are characterized by a finite cyclotron mass $m_{\mathrm{c}}$. Values for $m_{\mathrm{c}}$ can be extracted from the thermal damping of $\mathrm{SdH}$ oscillations via the same expression as in conventional 2D systems [38]: $R_{x x} \propto T / \sinh \left(2 \pi^{2} k T m_{\mathrm{c}} / \hbar e B\right)$. Experiments find the carrier-density dependence of $m_{\mathrm{c}}$ to be well described by:

$$
m_{\mathrm{c}}=E_{\mathrm{F}} / v_{\mathrm{F}}^{2}=\left(h^{2} n_{S} / 4 \pi v_{\mathrm{F}}^{2}\right)^{1 / 2} .
$$

Equation (6) is behind the use of $m_{\mathrm{c}}$ as the "relativistic" mass of Dirac fermions, where $v_{\mathrm{F}}$ plays the role of $c^{*}$. Here, $v_{\mathrm{F}}$ is found to be $\approx 1.0 \times$ $10^{6} \mathrm{~m} / \mathrm{s}$ experimentally $[13,14,34,35]$, in good agreement with band-structure calculations for $m_{\mathrm{c}} / m_{0}$ (Fig. 5b). As $E_{\mathrm{F}}$ approaches zero, i.e., the charge- 

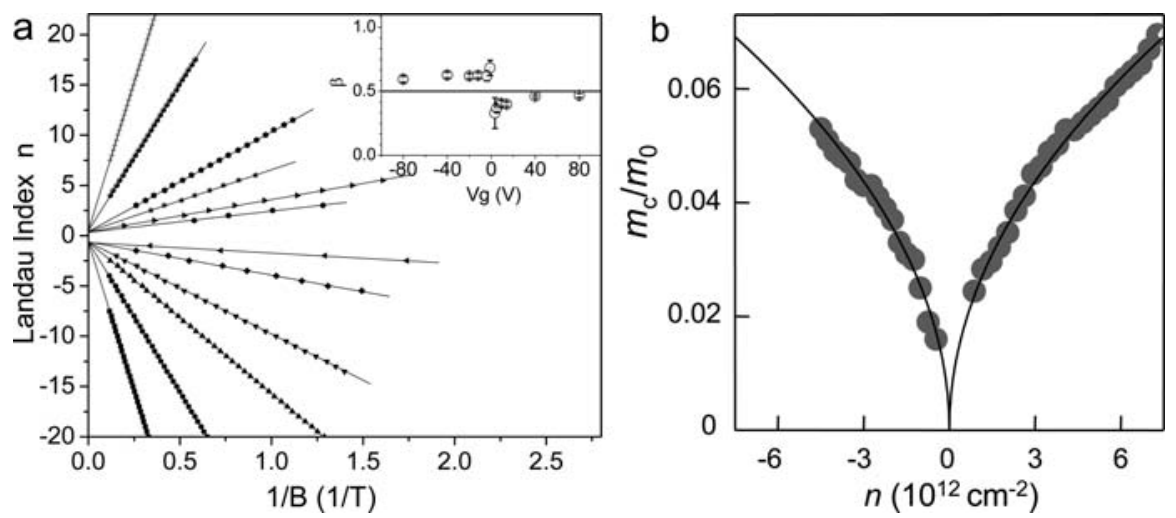

Fig. 5. Berry's phase $\pi$ and the cyclotron mass of Dirac fermions in single-layer graphene. (a) LL fan diagram plotting the positions of the resistance minima in $\mathrm{SdH}$ oscillations against the LL index $n$. Different lines correspond to different carrier densities produced by the backgate. The $n$-axis intercept yields Berry's phase $2 \pi \beta$. Analysis in single-layer graphene consistently yields $\beta=0.5$ (inset), providing convincing evidence for Dirac fermions. Adapted from [14]. (b) Cyclotron mass obtained from temperature-dependent low-field $\mathrm{SdH}$ oscillations. $m_{\mathrm{c}}$ scales with $\sqrt{n_{S}}$ and vanishes near the Dirac point. The theoretical fit using (6) (solid line) yields $v_{\mathrm{F}} \approx 1 \times 10^{6} \mathrm{~m} / \mathrm{s}$. Adapted from [13]. Values of $m_{\mathrm{c}}$ as low as $0.007 m_{0}$ (for $n_{S} \sim 2 \times 10^{11} / \mathrm{cm}^{2}$ ) have been reported [14]

neutrality point, $m_{\mathrm{c}}$ vanishes accordingly. Values of $m_{\mathrm{c}}$ as low as $0.007 m_{\mathrm{e}}$ $\left(n_{S} \sim 2 \times 10^{11} / \mathrm{cm}^{2}\right)$ have been reported [14].

Graphene in ultrahigh magnetic fields up to $45 \mathrm{~T}$ displays additional plateaus in $\sigma_{x y}$ at filling factors $\nu=0, \pm 1, \pm 4[39,40]$. Whereas the single-particle Zeeman splitting was identified as the origin of the $\nu= \pm 4$ plateaus [39], the nature of the $\nu=0, \pm 1$ states is still unclear. The underlying mechanisms of these quantum Hall states are the focus of many recent theoretical discussions (see a review by [41]), where a variety of interaction-driven ground states and novel charge and spin excitations have been predicted by combining quantum Hall physics with graphene's unique linear excitation spectrum and four-fold degeneracy. Reducing disorder in the present samples is crucial to the examination of these exciting ideas.

\subsection{Graphene Nanoribbons}

Among carbon nanostructures based on graphene, ribbons a few nanometers in width have also been extensively studied [42-54]. Since graphene nanoribbons (GNRs) are just geometrically terminated graphene, their electronic structure has been modeled by imposing appropriate boundary conditions (i.e., standing waves) on the Schrödinger equation within the present simple 
(a) zigzag edge

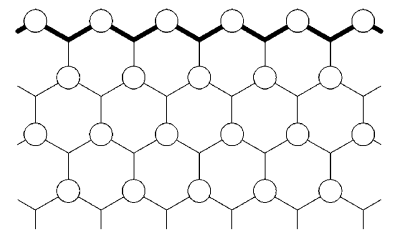

(b) armchair edge

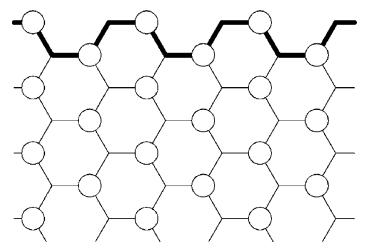

Fig. 6. GNR edges. (a) Zigzag edge, (b) armchair edge

tight-binding approximations based on the $\pi$-states of carbon [42, 43, 48] or on a two-dimensional Dirac equation with an effective speed of light $\left(c^{*} \sim 1 \times 10^{6} \mathrm{~m} / \mathrm{s}\right)[49-51]$.

Within these models, it is predicted that GNRs with "armchair" edges (AGNR) can be either metallic or semiconducting depending on their widths [42,43,48-51], and that GNRs with "zigzag" edges (ZGNR) are metallic with peculiar edge states on both sides of the ribbon regardless of their widths [4251] (Fig. 6). This edge state stems not from graphene nor from the dangling bonds at the edge, but from the topology of the $\pi$-electron networks across a significant part of the Brillouin zone and has no counterpart in an armchair edge. The corresponding energy bands are almost flat at the Fermi level, inducing a sharp peak in the density of states at $E_{\mathrm{F}}$. The charge density of the edge state is strongly localized on the zigzag edge sites. Although a general GNR is often characterized by a mixture of zigzag and armchair sites [42, 44], theory predicts that a GNR edge with three or four zigzag sites per sequence is sufficient to exhibit the characteristics of a zigzag edge state [42]. Indeed, STM and STS measurements [55] have observed evidence of this edge state near $E_{\mathrm{F}}$ at zigzag edge sites and at defect sites of armchair edges, but not at sites along a homogeneous armchair edge.

Although the tight-binding approximation based on $\pi$-states of carbon is known to accurately describe the energy dispersion of the carbon sheet, a careful consideration of edge effects in GNRs only a few nanometers in width is required to determine their band structures accurately [45, 48]. Recent ab-initio calculations [52-54] reveal that all GNRs with hydrogen-passivated armchair- or zigzag-shaped edges have nonzero direct bandgaps. The gap size decreases as the width of the GNR increases, approaching zero in graphene in the limit of infinite width. The origins of the bandgaps for the different types of homogeneous edges vary.

The bandgaps of GNRs with armchair edges originate from quantum confinement, but edge effects play a crucial role [52, 53]. Hydrogen passivation introduces $\mathrm{C}-\mathrm{H} \sigma$ bonds (or other types of terminations in general) at the edge, causing the $\mathrm{C}-\mathrm{C}$ bond length and the on-site energy of the $\mathrm{C}$ atoms at the armchair edge to be different from those in the interior of the GNR. The decrease of the $\mathrm{C}-\mathrm{C}$ bond length induces an increase of $\sim 12 \%$ in the hopping integral between $\pi$-orbitals. This physical deformation at the edge is 
responsible for the presence of a bandgap even for the armchair GNRs [52,53], which are predicted to be metallic in tight-binding calculations.

In the case of GNRs with zigzag edges, bandgaps arise upon the inclusion of the spin degrees of freedom [47,52-54]. The existence of the narrowband edge states at the Fermi level implies possible magnetization at the edges $[43,46,47,52-54]$. The difference in total energy per atom on the edge between non-spin-polarized and spin-polarized edge states is found to be of the order of a few tens of meV $[52,53]$. Spin-polarization effects in the electronic structure are discussed in the contribution to this volume by Spataru et al. Indeed, the zigzag GNRs are predicted to have a magnetic insulating ground state with ferromagnetic ordering at each zigzag edge and antiparallel spin orientation between the two edges. Because the two edge states with opposite spins occupy different sublattices, magnetic ordering leads to staggered sublattice potentials, which introduce bandgaps for electrons on a honeycomb hexagonal lattice [56]. Since the strength of the staggered potentials in the interior of the ribbon decreases as the ribbon width increases, the bandgaps of zigzag GNRs are inversely proportional to the width.

Similar to carbon nanotubes, optical spectra of GNRs are affected by selfenergy and many-body corrections to the single-particle excitation spectrum and are characterized by bound excitons, with binding energies $\sim 0.5-1 \mathrm{eV}$ for ribbons of a width comparable to the circumference of a typical SWNT [57]. The exciton binding energy scales inversely with the ribbon width [57].

Experimental exploration of GNRs is at a very early stage. Recently, GNRs with $10-500 \mathrm{~nm}$ width have been patterned lithographically using hydrogen silsesquioxane as an etch mask and oxygen plasma etching [58,59]. Such an approach most likely produces mixed zigzag and armchair edges terminated by carboxyl groups. It was shown by Han et al. [58] that lateral confinement indeed leads to energy gaps with a ribbon width $W$ dependence given by $E_{\text {gap }}=\alpha /\left(W-W^{*}\right)$, where $\alpha=0.2 \mathrm{eV} \mathrm{nm} \mathrm{and} W^{*}=16 \mathrm{~nm}$ represents the offset in the active ribbon width determination. This empirical relation is consistent with DFT calculations presented in $[52,53]$. Furthermore, unlike the closed structure of carbon nanotubes, the dangling bonds at the edges of a GNR may be chemically functionalized to perform selective chemical/biological sensing. Edge functionalisation and atomic substitutions can indeed modify the electronic and spin properties of the GNRs [60]. This whole new direction awaits experimentation.

In conclusion, the role of the edges is crucial in determining the values and scaling rules for the bandgaps in both armchair and zigzag GNRs. In addition, although the spin-orbit interaction is very small in graphite [61], the coexistence of electrons with opposite spin orientations in GNRs could open up a new path to the exploration of spintronics at the nanometer scale (when the electrical current is completely spin polarized) [54]. The ability to selectively create specific edge types on demand is essential to test GNR theories and evaluate GNR-based devices. 


\subsection{Graphite and $n$-Graphene Layer Systems}

Bulk graphites consist of a tridimensional (3D) stacking of graphene sheets with an interlayer spacing around 3.35-3.4 A. However, various graphites differ in the stacking sequence of the carbon planes, such as simple hexagonal graphite $(A A A \ldots)$; Bernal graphite $(A B A B \ldots)$; and rhombohedral graphite $(A B C A B C \ldots)$. These graphene-based $3 \mathrm{D}$ crystals exhibit specific semimetallic properties that depend directly on the geometry of the stacking between layers [62-65]. Consequently, the nature of the charge carriers strongly depends on the particular stacking of the graphene sheets and the corresponding Fermi surfaces exhibit a complex shape around the $H-K-H$ line at the edge of the 3D hexagonal Brillouin zone [62-65].

In contrast to the corresponding $3 \mathrm{D}$ bulk structures, electrons in an $n$ graphene layer ( $n \mathrm{GL}, n=$ a small integer) are confined along the crystallographic direction perpendicular to the graphene sheets, offering a genuine 2D character to the $n$ GL electronic states. However, the linear dispersion of the electronic bands near the Fermi level that appears in monolayer graphene is lost, as a consequence of the interaction between layers.

Recent ab-initio electronic calculations [66] have revealed that $n$ GLs belong to an intermediate case between bulk graphite and a graphene sheet. The corresponding band structures of $n$ GLs will be reminiscent of both cases. The weak interlayer interaction that creates the $c$-axis band dispersion in $3 \mathrm{D}$ graphites is now responsible for band mixing of states otherwise associated with isolated graphene bands. The number of layers and the stacking order are key factors influencing the transport properties of $n$ GLs $[66,67]$. Indeed, depending on these factors, an $n \mathrm{GL}$ can behave as a 2D metal (with a single or mixed-carrier type) or a narrow-gap 2D semiconductor. For example, amongst 3- and 4-layer $n \mathrm{GLs}, A B A C$ stacking is found to lead to a narrow-gap semiconductor (with a bandgap $\sim 9 \mathrm{meV}$ ) [66]. Other stackings can exhibit a metallic behavior with different signatures: the rhombohedral family $(A B C, A B C A)$ displays a very small $(<3 \mathrm{meV})$ overlap between electron and hole bands, while the Bernal family $(A B A, A B A B)$ shows overlaps of a few tens of meV (from $5-20 \mathrm{meV}$ ) in agreement with experimental measurements [66]. Besides perfectly stacked ABAB structures, $n$ GLs might also be made to exhibit a turbostratic random stacking, leading to a weak band overlap [66,68].

Among $n$ GLs, the simplest and arguably most interesting case may be bilayer graphene (2GL). A calculated band structure of $2 \mathrm{GL}$ is given in Fig. 7a together with angle-resolved photoemission spectroscopy (ARPES) measurements by Ohta et al. [11]. A gap of $\sim 0.8 \mathrm{eV}$ appears for the high-energy bands due to the interlayer interaction. This splitting is predicted to be independent of carrier concentration in the two layers [69]. The splitting of the low-energy bands, however, arises from the broken inversion symmetry of the two constituent layers and sensitively depends upon the strength of the interlayer electric field. The experiments of Ohta et al. [11] used potassium 
a

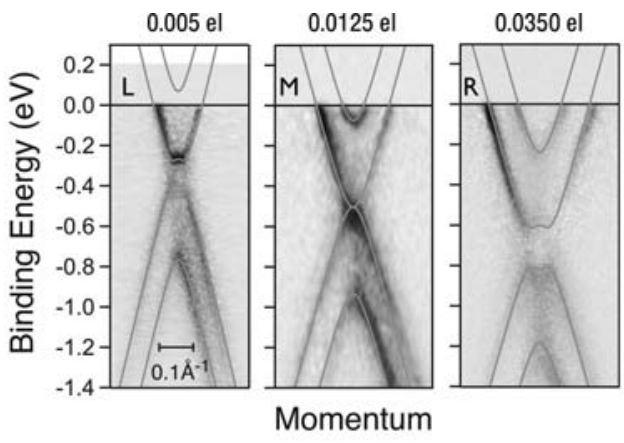

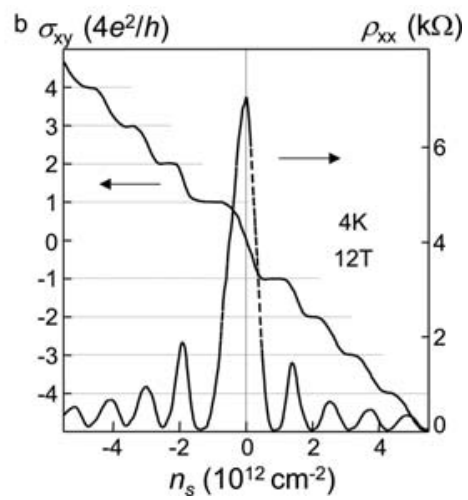

Fig. 7. Band structure and quantum Hall effect of bilayer graphene. (a) Band structure measured by angle-resolved photoemission spectroscopy. From left to right: Tuning the bandgap of the low-energy bands by increasing the amount of potassium doping. Calculations [69] are shown for comparison (solid lines). Adapted from [11]. (b) Quantum Hall effect in bilayer graphene showing quantized steps of $\sigma_{x y}$ at $4 n e^{2} / h . \sigma_{x y}$ jumps from $-4 e^{2} / h$ to $4 e^{2} / h$ across the charge-neutrality Dirac point. Adapted from [28]

atoms to continuously and preferentially dope the top layer of the bilayer graphene, allowing a continuous tuning of the gap opening in the low-energy bands. The resulting band structures are shown in Fig. 7a. More recently, Castro et al. [70] demonstrated that by combining chemical doping and fieldeffect doping imposed by a backgate, the gap splitting and the Fermi level of bilayer graphene can be independently controlled. This gap-tuning capability may have important implications in the development of graphene-based electronics.

Bilayer graphene (2GL) with parabolic but degenerate bands at the Dirac point (middle panel of Fig. 7a) exhibits a distinctive quantum Hall effect. The LLs of these massive, chiral quasiparticles are described by [69]:

$$
E_{n}=\frac{\hbar e B}{m^{*}} \sqrt{n(n-1)}
$$

The conventional integer quantum Hall sequence $\sigma_{x y}=4 n e^{2} / h$ is restored for the 2GL. However, the merging of the $n=1$ and the $n=0$ LLs leads to an eightfold degeneracy at the charge-neutrality point and the absence of the $\nu=0$ plateau $\sigma_{x y}$. These features are signatures of a bilayer graphene sample and have been observed recently by Novoselov et al. [28] (Fig 7b). 

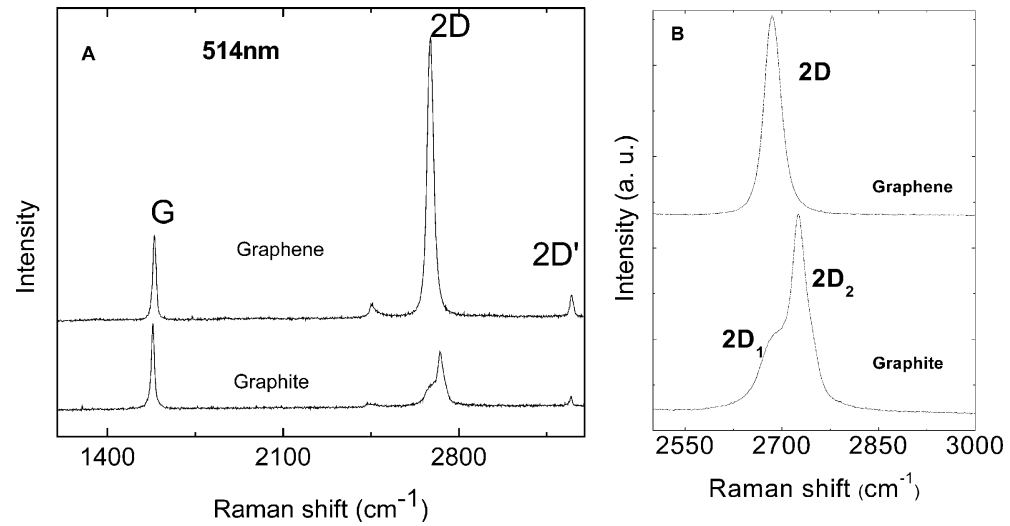

Fig. 8. (A) Comparison of the Raman spectra of graphene and graphite measured with 514.5-nm excitation. (B) Comparison of the 2D peaks in graphene and graphite (also measured with 514.5-nm excitation)

\section{Optical Phonons and Raman Spectroscopy}

Presently, the widely used micromechanical cleavage method produces $n \mathrm{GL}$ flakes with varying $n$ and the yield of low- $n$ graphene flakes is low. A simple method to locate these flakes and determine the number of layers is much needed for the studies of these materials. $n$ GLs are optically invisible on most substrates. Their optical detection requires an optimal combination of detection wavelength and oxide thickness [71] and so far all the successful observations of single- and bilayer graphene are made with Si substrates with a specific oxide thickness of $\sim 300 \mathrm{~nm}[7]$. Alternatively, an atomic force microscope (AFM) may be used to identify $n$ GLs. This approach has two drawbacks. First, AFM suffers from low throughput. Secondly, instead of the expected graphite interlayer spacing $0.34 \mathrm{~nm}$, AFM height measurements report an apparent step height of $0.5-1 \mathrm{~nm}$ between a graphene flake and the $\mathrm{SiO}_{2}$ substrate due to their difference in chemical composition. This variable offset can present problems for the AFM identification of 1GL and 2GL films. Here, we show that an $n$ GL's electronic structure is uniquely captured in its Raman spectrum. Raman fingerprints for single-, bi- and few-layer graphene allow unambiguous, high-throughput, nondestructive identification of the number of layers.

\subsection{Raman D and G Bands, Double Resonance and Kohn Anomalies}

Figure 8 compares the Raman spectra of graphene and bulk graphite collected with 514-nm (2.41 eV) excitation [72]. In general, the main features in the first order Raman spectra of graphitic carbons are the so-called G and D bands, 
which lie at $\sim 1580$ and $\sim 1360 \mathrm{~cm}^{-1}$, respectively, for visible excitation [73]. The assignment of these bands is straightforward in the "molecular" picture of carbon materials. They are present in all polyaromatic hydrocarbons [74, 75]. The $\mathrm{G}$ band is due to the bond stretching of all pairs of $s p^{2}$-bonded atoms in both rings and chains. The $\mathrm{D}$ band is due to the breathing modes of $s p^{2}-$ bonded atoms in rings $[74,76,77]$. However, this molecular approach to the interpretation of these Raman bands in graphitic carbons has undergone a debate that has lasted several decades. The $\mathrm{D}$ band was first attributed to a breathing mode for phonons with wavevectors near the $\left(K, K^{\prime}\right)$ points, activated by disorder (D). This relaxes the fundamental Raman selection rule, which states that only phonons near the $\Gamma$ point can be seen in first order Raman scattering [76]. The D band was then linked to maxima in the vibrational density of states of graphite at the $M$ and $K$ points of the Brillouin zone $[78,79]$. However, this does not account for the dispersion of the Raman D-peak position with photon excitation energy [80,81], or why the D-peak overtone ( $2 \mathrm{D}$ band) at $\sim 2710 \mathrm{~cm}^{-1}$ can be observed even in the absence of the $\mathrm{D}$ peak, or why the intensity ratio $I(\mathrm{D}) / I(\mathrm{G})$ is dispersive (i.e., a function of the incident photon energy) [80,81]. Phonon confinement does not explain why the $\mathrm{D}$ mode is more intense than other modes closer to $\Gamma$, nor why it is seen in disordered graphite with an in-plane correlation length, $L_{a}$, as large as $30 \mathrm{~nm}$ [82].

Pocsik et al. [81] proposed that the D band arises due to a resonant Raman coupling in which there is a strong enhancement of the Raman cross section of a phonon of wavevector $\boldsymbol{q}$, when $\boldsymbol{q}=\boldsymbol{k}$, the wavevector of the vertical electronic transition excited by the incident photon ("quasi-selection rule") [77]. However, this "quasi-selection rule" does not explain why, amongst all phonons satisfying this condition, only those on one particular optical branch are seen. Thomsen and Reich [83] and Baranov et al. [84] proposed double resonance (DR) as the activation mechanism. Within DR, Raman scattering is a fourth-order process involving four virtual transitions: 1. excitation of an electron-hole pair; 2. electron-phonon scattering with an exchanged momentum $\boldsymbol{q} \sim \boldsymbol{K}$, where $\boldsymbol{K}$ is the $K$ point Brillouin zone vector; 3 . defect scattering; 4 . electron-hole recombination. The DR condition is reached when the energy is conserved over all these four transitions [83]. A similar "intravalley" process is possible. This activates phonons with a small $\boldsymbol{q}$, resulting in the so-called $\mathrm{D}^{\prime}$ peak, that appears at $\sim 1620 \mathrm{~cm}^{-1}$ in graphite with defects [78].

The phonon dispersion around the $\mathrm{K}$ point is also crucial for the correct interpretation of the Raman $\mathrm{D}$ band. Graphene has four frequencies at the $K$ point, originating from six phonon branches around $K$ (three are shown in Fig. 9 that focus of the longitudinal optical (LO) and transversal optical (TO); a fourth lower-lying optical branch crossing the $K$ point at $\sim 800 \mathrm{~cm}^{-1}$ also exists). All these branches should be Raman active if their electron-phonon couplings (EPCs) are ignored [81, 83, 86-88]. However, only a single D band is observed [76]. In the molecular approach $[74,75,77,89]$, the 


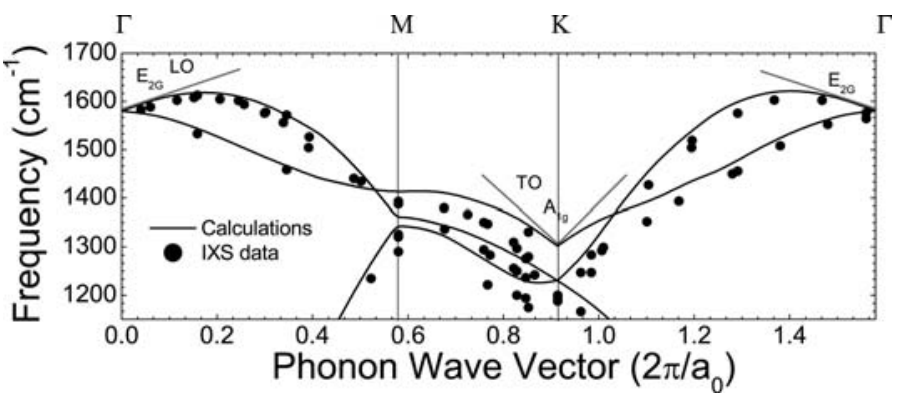

Fig. 9. Phonon dispersion of graphene calculated by DFPT [18] compared with the inelastic X-ray scattering data of [85]. The lines emphasize the presence of the two Kohn anomalies at $\boldsymbol{\Gamma}$ and $\boldsymbol{K}$

D band was assigned to the highest optical branch near $\boldsymbol{K}$. This assignment is based on symmetry and on the large Raman cross section of large aromatic molecules. However, this assignment was initially disputed [81, 83, 8688]. In fact, the measured linear $\mathrm{D}$ band dispersion with excitation energy [81] seemed at odds with the flat, or even negative, slope of the highest optical branch near $K$ given by previous calculations [90-94]. Many authors $[81,83,86-88]$ therefore initially attributed the D band to the doubly degenerate, linearly dispersive $1200 \mathrm{~cm}^{-1} \mathrm{E}^{\prime}$ mode at the $K$ point. Piscanec et al. [18] finally confirmed the attribution of the $\mathrm{D}$ band to the highest optical $\left(\mathrm{A}_{1}\right)$ branch near $K\left(\boldsymbol{K}-A_{1}^{\prime}\right)[76,77,89,90]$. Indeed, theoretical calculations have shown that the $A_{1}^{\prime}$ branch has by far the biggest EPC amongst all the $K$ point phonons [20]. Secondly, the $\boldsymbol{K}-A_{1}^{\prime}$ branch exhibits linear dispersion near the $K$ point (Fig. 9). A Kohn anomaly at $K$ is the physical origin of this linear dispersion, which is in quantitative agreement with the measured D-band dispersion [81].

In general, atomic vibrations are partially screened by filled electronic states. In a metal, this screening is determined by the shape of the Fermi surface and can change rapidly from one point to another in the Brillouin zone (BZ). The consequent anomalous behavior of the phonon dispersion is called a Kohn anomaly [95]. Kohn anomalies may occur only for phonon wavevectors $\boldsymbol{q}$ such that there are two electronic states $\boldsymbol{k}_{\mathbf{1}}$ and $\boldsymbol{k}_{\mathbf{2}}$ on the Fermi surface, where $\boldsymbol{k}_{\mathbf{2}}=\boldsymbol{k}_{\mathbf{1}}+\boldsymbol{q}$ [95]. In graphene, the gap between occupied and empty electronic states is zero at the six corners of the BZ $\left(\boldsymbol{K}\right.$ and $\left.\boldsymbol{K}^{\prime}\right)$. These points are connected by a vector of length $K$. Thus, Kohn anomalies can occur for $\boldsymbol{q}=0(\Gamma)$ or $\boldsymbol{q}=\boldsymbol{K}$ (Fig. 9 [18]). The cusps in $\omega(\boldsymbol{q})$ calculated at $\boldsymbol{q}=0$ or $\boldsymbol{q}=\boldsymbol{K}$ cannot be described by a finite set of interatomic force constants [18]. Interestingly, for a given value of $\boldsymbol{q}$, the Kohn anomalies are present only in the highest optical branches. This can be understood from the details of the EPC [18-21]. 


\subsection{Electron-Phonon Coupling from Phonon Dispersions and Raman Linewidths}

Electron-phonon coupling (EPC) is important to the properties of graphene and nanotubes. Ballistic transport, superconductivity, excited-state dynamics, Raman spectra and phonon dispersions all fundamentally depend on it. In nanotubes, the EPC of the optical phonon is also extremely relevant, since electron scattering by optical phonons sets the ultimate limit to high-field ballistic transport [21,96-99]. Many contradicting tight-binding calculations of EPC for optical phonons in graphene and nanotubes are in the literature [97, 100-103] (see Table II of Ref. [19] for a summary).

References $[18,20]$ presented DFT calculations of graphene optical phonons and the EPC, as well as a strategy for their experimental determination. The presence of the Kohn anomalies is revealed by two sharp kinks in the phonon dispersion $\omega(\boldsymbol{q})$ (Fig. 9). Their slope $S$ is proportional to the ratio of the square of the EPC and the electronic $\pi$ band slope $\beta$ [18]:

$$
S_{\Gamma}^{\mathrm{LO}}=\frac{\sqrt{3} \hbar a_{0}^{2}}{8 M \omega_{\Gamma} \beta} \operatorname{EPC}(\Gamma)^{2},
$$

and

$$
S_{K}^{\mathrm{TO}}=\frac{\sqrt{3} \hbar a_{0}^{2}}{8 M \omega_{\boldsymbol{K}} \beta} \operatorname{EPC}(K)^{2},
$$

where $\beta=\hbar v_{\mathrm{F}}=5.52 \AA \mathrm{eV}, M$ is the carbon atomic mass, and $\omega_{\boldsymbol{\Gamma}}$ and $\omega_{\boldsymbol{k}}$ are, respectively, the frequency of the $\mathrm{E}_{2 g}$ phonon at $\Gamma$ and the $\boldsymbol{K}-A_{1}^{\prime}$ phonon. The experimental phonon dispersion therefore can be used to measure the EPC [18-20].

An alternative strategy for the EPC measurement is based on the analysis of the G-peak linewidths. The EPC is the major source of broadening for the Raman $\mathrm{G}$ band in graphite, graphene and for the $\mathrm{G}^{-}$peak in metallic nanotubes $[19,20]$.

In a perfect crystal, the Raman linewidth $\gamma$, or inverse phonon lifetime, is determined by the anharmonic terms in the interatomic potential and the EPC, i.e., $\gamma=\gamma^{\text {an }}+\gamma^{\mathrm{EPC}}$, in which $\gamma^{\text {an }}$ is always present and $\gamma^{\mathrm{EPC}}$ is important only in metals since the carrier density interacting with phonons would be very low for semiconductors. If $\gamma^{\text {an }}$ is negligible or otherwise known, measuring the linewidth is a simple way to determine the contribution from EPC. This is the case in graphene, graphite and metallic carbon nanotubes, where $\gamma^{\text {an }}$ is much smaller than $\gamma^{\text {EPC }}[20]$.

From Fermi's golden rule, $\gamma_{0}^{\mathrm{EPC}}$ can be described by a simple analytical formula [20]:

$$
\gamma_{0}^{\mathrm{EPC}}=\frac{\sqrt{3} \hbar^{2} a_{0}^{2}}{4 M \beta^{2}}[\operatorname{EPC}(\Gamma)]^{2},
$$


provided that the conservation of energy and momentum is fulfilled (i.e., $\left.\boldsymbol{q} \leq \hbar \omega_{\boldsymbol{\Gamma}} / \beta\right)$. Otherwise, $\gamma_{0}^{\mathrm{EPC}}=0$. This condition is satisfied by the Raman $\mathrm{G}$ peak of undoped graphite and graphene. On the other hand, the doubleresonant $\mathrm{D}^{\prime}$ mode close to $\mathrm{G}$ does not satisfy this criterion. Indeed, the $\mathrm{D}^{\prime}$ peak is sharper than the G peak [104]. The experimental G bandwidth full width at half-maximum $(\operatorname{FWHM}(\mathrm{G}))$ of graphite and undoped graphene is $\sim 13 \mathrm{~cm}^{-1}[20,105]$. Temperature-dependent measurements show no increase of $\mathrm{FWHM}(\mathrm{G})$ in the $2-900 \mathrm{~K}$ range [105]. Accounting for a Raman spectrometer resolution of $\sim 1.5 \mathrm{~cm}^{-1}$, this implies that the anharmonic contribution is lower than the spectral resolution. Thus, $\gamma_{0}^{\mathrm{EPC}}(\mathrm{G}) \sim 11.5 \mathrm{~cm}^{-1}$. Then, from $(10),[E P C(\Gamma)]^{2} \sim 47(\mathrm{eV} / \AA)^{2}$. This compares very well with DFT calculations $[19,20]$, further supporting a small $\gamma^{\text {an }}$.

Finally, [17, 106] extended (10) to finite doping $\left(\epsilon_{\mathrm{F}} \neq 0, \epsilon_{\mathrm{F}}\right.$ being the Fermi level):

$$
\gamma^{\mathrm{EPC}}=\gamma_{0}^{\mathrm{EPC}}\left[f\left(-\frac{\hbar \omega_{0}}{2}-\epsilon_{\mathrm{F}}\right)-f\left(\frac{\hbar \omega_{0}}{2}-\epsilon_{\mathrm{F}}\right)\right],
$$

where $f(E)$ is the Fermi-Dirac distribution. Note that, even for zero doping, (11) predicts a significant decrease of $\gamma^{\mathrm{EPC}}$ with temperature. Since the anharmonic contribution to the FWHM is much smaller than $\gamma^{\mathrm{EPC}}$, (11) predicts a net decrease of $\mathrm{FWHM}(\mathrm{G})$ with temperature, in contrast to what happens in most materials. This unusual trend was indeed observed recently [105].

\subsection{The Raman Spectrum of Graphene and $n$-Graphene Layer Systems}

Figure 8 shows that the two most intense features in the Raman spectrum of graphene are the $\mathrm{G}$ peak at $\sim 1580 \mathrm{~cm}^{-1}$ and a band at $\sim 2700 \mathrm{~cm}^{-1}$, historically named $\mathrm{G}^{\prime}$, since it is the second most prominent band always observed in graphite samples [80]. However, we now know that the $\mathrm{G}^{\prime}$ band is due to second-order Raman scattering at $\omega=\omega_{1}(\boldsymbol{q})+\omega_{2}(-\boldsymbol{q})$, where $\boldsymbol{q} \sim \boldsymbol{K}$ and $\omega_{1}=\omega_{2}=\omega_{D}$. Thus, we refer to the $\mathrm{G}^{\prime}$ band as the $2 \mathrm{D}$ band. Figure 8 also shows another peak at $\sim 3250 \mathrm{~cm}^{-1}$. Its frequency is higher than double the G-peak frequency, thus it is not the second-order scattering of $\mathrm{G}$ phonons. This peak has been identified with the second-order scattering of the intravalley $\mathrm{D}^{\prime}$ band discussed above. Thus, for consistency, we call it the $2 \mathrm{D}^{\prime}$ band. Figure $8 \mathrm{~b}$ shows a significant change in the shape and intensity of the $2 \mathrm{D}$ band of graphene compared to bulk graphite. The $2 \mathrm{D}$ band in bulk graphite consists of two components $2 \mathrm{D}_{1}$ and $2 \mathrm{D}_{2}[78,80]$, roughly $1 / 4$ and $1 / 2$ of the intensity of the $\mathrm{G}$ band, respectively. Graphene has a single, sharp $2 \mathrm{D}$ peak, roughly 4 times more intense than the $\mathrm{G}$ peak.

Figure 10 shows the evolution of the 2D band in $n$ GLs as a function of the number of layers for $514.5 \mathrm{~nm}$ excitation [72]. Bilayer graphene $(n=2)$ 

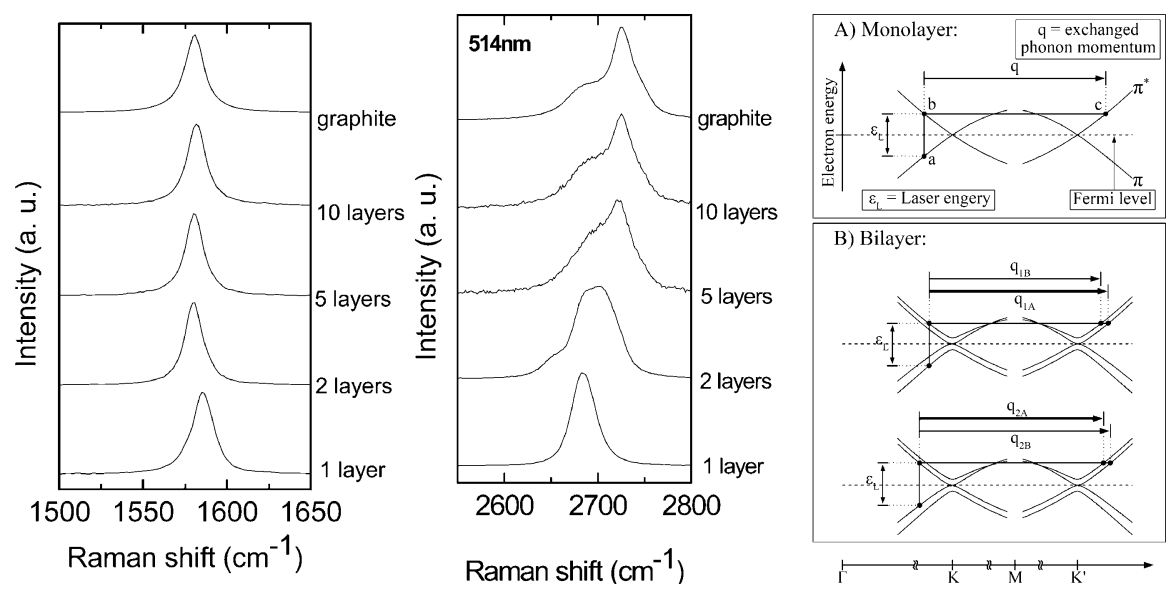

Fig. 10. Left and Center: Raman spectra for the G band (left) and for the 2Dband (right) of $n$ GL with varying $n$. Right: The DR process for the $2 \mathrm{D}$ peak in (A) graphene and (B) bilayer graphene

has a much broader and upshifted 2D band with respect to graphene. The shape of this band is quite different from that of bulk graphite. It can be well fitted by 4 components, $2 \mathrm{D}_{1 \mathrm{~B}}, 2 \mathrm{D}_{1 \mathrm{~A}}, 2 \mathrm{D}_{2 \mathrm{~A}}, 2 \mathrm{D}_{2 \mathrm{~B}}$, two of which, $2 \mathrm{D}_{1 \mathrm{~A}}$ and $2 \mathrm{D}_{2 \mathrm{~A}}$, are more intense than the other two, as indicated in Fig. 11. Increasing the number of layers further leads to a significant decrease of the relative intensity of the lower-frequency $2 \mathrm{D}_{1}$ peaks. The Raman spectrum of an $n \mathrm{GL}$ of more than 5 layers becomes hardly distinguishable from that of bulk graphite [72]. In contrast, the shape of the $\mathrm{G}$ band does not change with the number of layers. However, a slight frequency upshift can be seen in the case of graphene. This is due to a combination of self-doping [17], as discussed later, and the fact that the frequency of the Raman active phonon in graphene is slightly higher than in graphite, due to mode splitting (see lower panel of Fig. 1 in [18]).

Figure 12 shows the effect of laser excitation energy on the shape and position of the 2D band [107]. Spectra for $n=1-4$ are shown in three panels corresponding to excitations at $785 \mathrm{~nm}$ (left), $514.5 \mathrm{~nm}$ (middle) and $488 \mathrm{~nm}$ (right). These spectra are fitted to four Lorentzian components $\left(2 \mathrm{D}_{1 \mathrm{~A}}, 2 \mathrm{D}_{1 \mathrm{~B}}\right.$, $\left.2 \mathrm{D}_{2 \mathrm{~A}}, 2 \mathrm{D}_{2 \mathrm{~B}}\right)$ as described above. The relative intensities of these components within the 2D manifold change with laser excitation energy. The simple, nearLorentzian lineshape of the $n=1$ band is preserved and a shift in peak position of $\sim 100 \mathrm{~cm}^{-1} / \mathrm{eV}$ with increasing excitation energy is observed [72, 83, 89, 108].

Figure 13 shows the peak-position shift with layer number $n$ for the 3 rdorder Raman band near $4270 \mathrm{~cm}^{-1}$ [109]. The spectra were collected with a 514.5-nm excitation and the $\sim 4270 \mathrm{~cm}^{-1}$ band is identified with a three- 


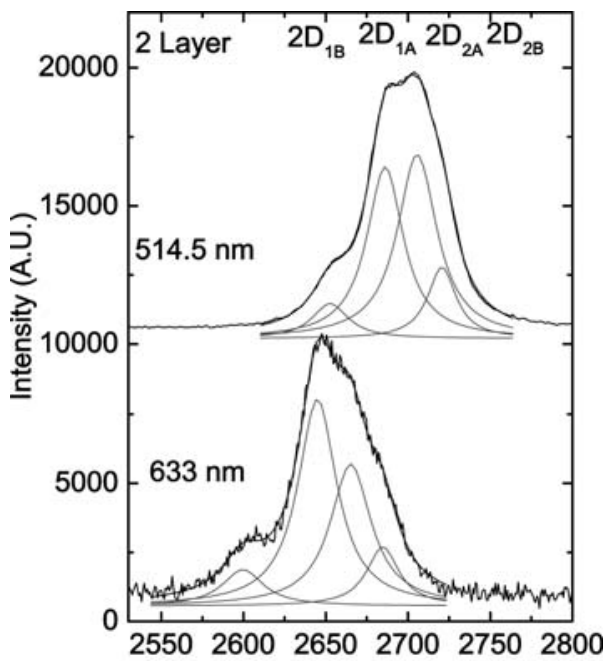

Fig. 11. The four components of the $2 \mathrm{D}$ peak in bilayer graphene at two laser-excitation waveRaman Shift $\left(\mathrm{cm}^{-1}\right)$ lengths [72]
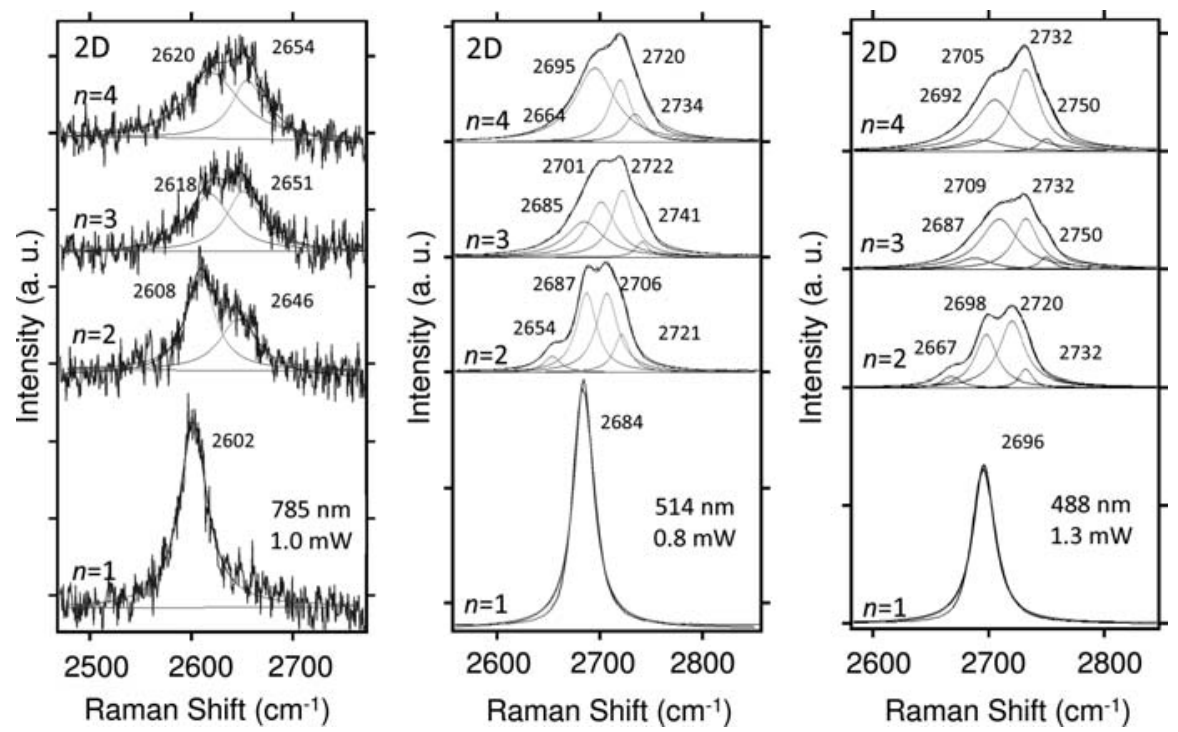

Fig. 12. Excitation dependence of the $2 \mathrm{D}$ band for $n=1-4 n$ GLs supported on $\mathrm{SiO}_{2}: \mathrm{Si}$ 

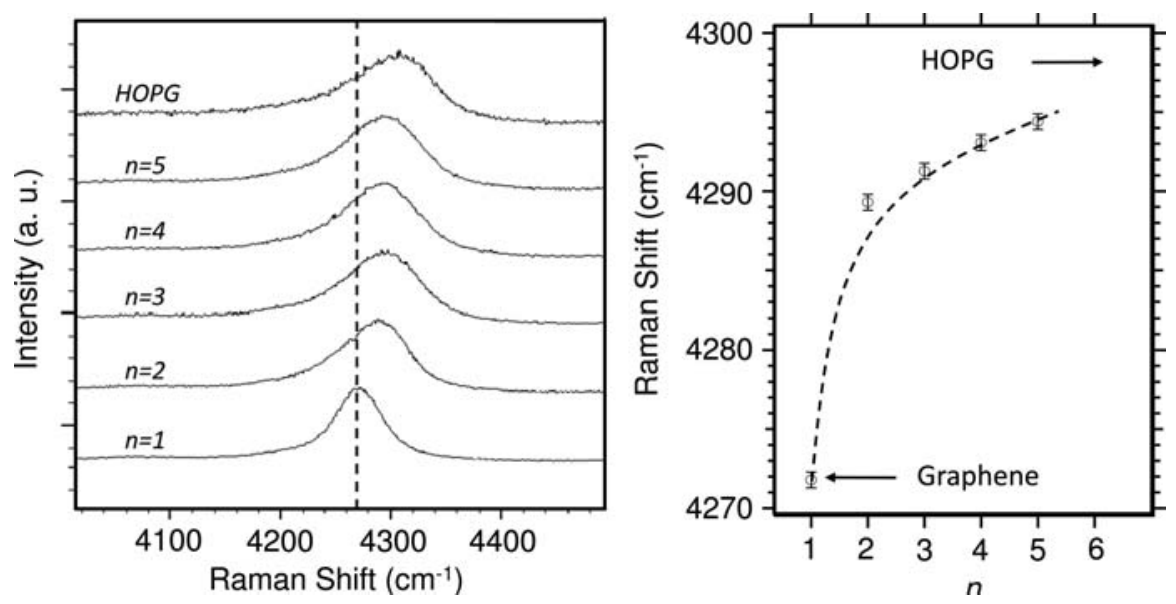

Fig. 13. 3rd-order Raman bands assigned to $2 \mathrm{D}+\mathrm{G}$ phonons. Left: $\mathrm{HOPG}$ and $n=1-5$ supported on $\mathrm{SiO}_{2}$ :Si. Right: Band position vs. $n$. Data collected with 514.5-nm excitation

phonon process in which $2 \mathrm{D}$ phonons $\left(\sim 2 \times 1350 \mathrm{~cm}^{-1}\right)$ and one $\mathrm{G}$ phonon $\left(\sim 1580 \mathrm{~cm}^{-1}\right)$ are created simultaneously in the scattering process. We call this band $2 \mathrm{D}+\mathrm{G}$. The left panel shows the $n$ dependence of the lineshape and the right panel displays the $n$ dependence of the band maximum. As can be seen in the left panel, graphene exhibits a nearly symmetric $2 \mathrm{D}+\mathrm{G}$ band that asymmetrically broadens to lower frequency and upshifts with increasing $n$. The shape of the band remains simple in character. This 3rd-order band appears to afford a means to determine the number of layers in the $n$ GL (as shown in the right panel). Therefore, for low $n$, the frequency rises steeply and then crosses over to an almost linear upshift with further increases in $n$ (the dashed curve in the figure is a guide to the eye).

The excitation-energy dependence of the 3rd-order $(2 \mathrm{D}+\mathrm{G})$ band is shown in Fig. 14 for 514-nm (left panel) and 488-nm (right panel) excitation. In both panels, the 3rd-order Raman band shifts to higher frequency with increasing layer number. The band dispersion of $93 \mathrm{~cm}^{-1} / \mathrm{eV}$ with excitation energy is consistent with its identification as a 3 -phonon $2 \mathrm{D}+\mathrm{G}$ process. That is, the frequency upshift with increasing excitation energy tracks that of the 2ndorder $2 \mathrm{D}$ band (the G-band remains fixed, independent of excitation). Other overtones up to $\sim 6000 \mathrm{~cm}^{-1}$ were also identified [110].

Ferrari et al. [72] explained why graphene (1GL) has a single 2D band, and why this band splits into four components in bilayer graphene and evolves into two distinguishable components in bulk graphite (see Fig. 10b). The 2D Raman band in graphene is due to two phonons with opposite momenta in the highest optical branch near the $K$ point [18, 76, 77]. The resulting 2D frequency is twice that of the scattering phonons, whose $\boldsymbol{q}$ is determined 

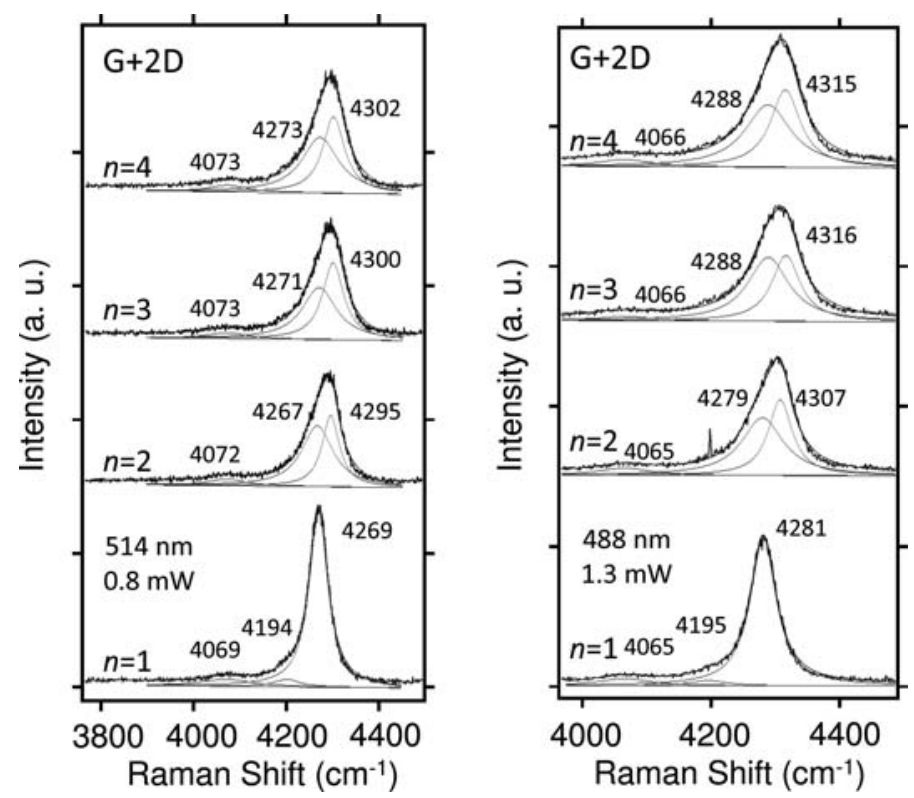

Fig. 14. Excitation dependence of the $2 \mathrm{D}+\mathrm{G}$ band for $n=1-4$. $n \mathrm{GLs}$ supported on $\mathrm{SiO}_{2}: \mathrm{Si}$

by the DR condition (Fig. 10B). In bilayer graphene, the interlayer interaction causes the $\pi$ and $\pi^{*}$ bands to divide into two hole and two electron bands, with a different splitting for electrons and holes (Fig. 10B). Amongst the 4 possible optical transitions, the incident light couples more strongly the two transitions shown in Fig. 10B. The two almost degenerate phonons in the highest optical branch couple all electron bands amongst them. The resulting four scattering processes involve phonons with momenta $\boldsymbol{q}_{1 \mathrm{~B}}, \boldsymbol{q}_{1 \mathrm{~A}}, \boldsymbol{q}_{2 \mathrm{~A}}$, and $\boldsymbol{q}_{2 \mathrm{~B}}$, as shown in Fig. 10B. These wavevectors correspond to phonons with different frequencies, due to the strong phonon dispersion around $\boldsymbol{K}$ [18]. They produce four different peaks in the Raman spectrum of bilayer graphene [72].

\subsection{Doped Graphene:}

\section{Breakdown of the Adiabatic Born-Oppenheimer Approximation}

Doping moves $\epsilon_{\mathrm{F}}$ and changes the Fermi surface of graphene. The Kohn anomaly then moves away from $\boldsymbol{q}=0$. Thus, since first-order Raman scattering probes $\boldsymbol{q}=0$ phonons, intuitively we might expect the G peak to stiffen with electron or hole doping. Indeed, this response is reported in $[17,111]$. There, the graphene sheet was supported on a $\mathrm{SiO}_{2}: \mathrm{Si}$ substrate and the doping level was controlled by applying a gate voltage. The $\mathrm{G}$ peak upshifts for both hole and electron doping. Figure 15 plots the G-peak position 

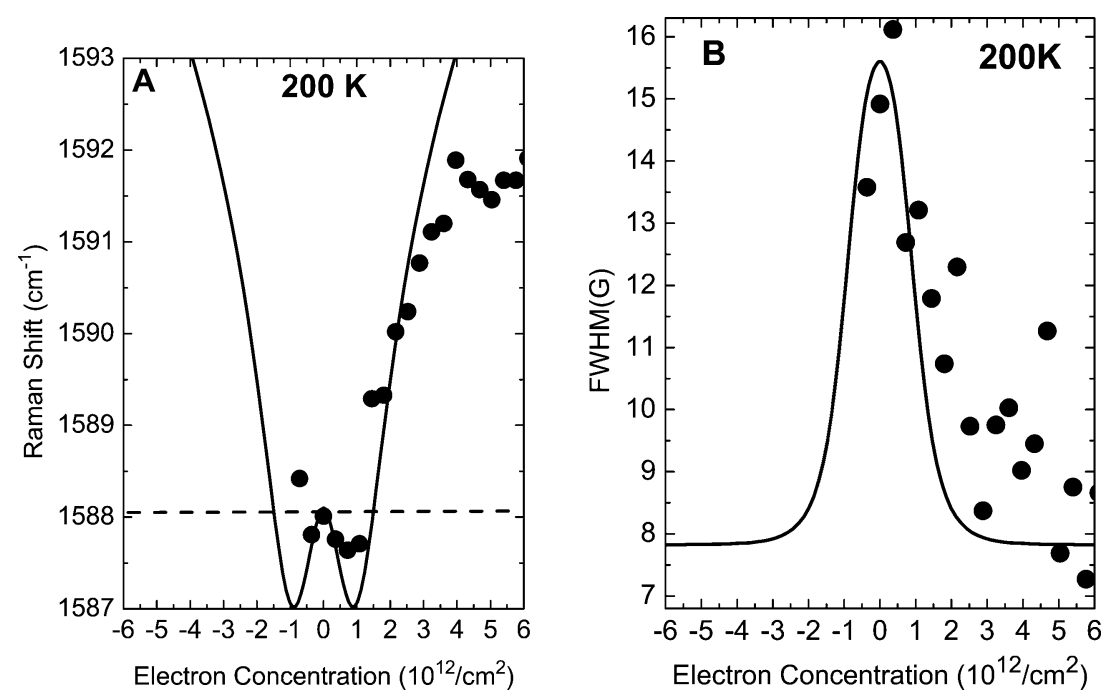

Fig. 15. (A) G peak position as a function of electron concentration at $200 \mathrm{~K}$. (dots) measurements; (horizontal-dashed line) adiabatic Born-Oppenheimer; (line) finite-temperature nonadiabatic calculation. The minimum observed in the calculations at $\sim 10^{12} / \mathrm{cm}^{2}$ occurs when the Fermi energy equals half of the phonon energy. (B) $\operatorname{FWHM}(\mathrm{G})$ at $200 \mathrm{~K}$ as a function of electron concentration. (dots) measurements; (line) theoretical FWHM of a Voigt profile obtained from a Lorentzian component given by (11), and a constant Gaussian component of $\sim 8 \mathrm{~cm}^{-1}$ [17]

and FWHM measured at $200 \mathrm{~K}$ as a function of electron doping. The trends in Fig. 15 are similar to those reported in [111] at $10 \mathrm{~K}$.

The trend in the FWHM is fully consistent with the prediction of (11). The upshift of the $\mathrm{G}$ peak with doping can be understood by extending the DFT calculations of [18] to include beyond-Born-Oppenheimer corrections to the dynamical matrix [17]. The detailed theoretical analysis of this case is reported in $[106,112,113]$.

The adiabatic Born-Oppenheimer approximation (ABO) has been standard in describing the interaction between electrons and nuclei since the early days of quantum mechanics [16]. ABO assumes that the lighter electrons adjust adiabatically to the motion of the heavier nuclei, remaining in their instantaneous ground states. ABO is well justified when the energy gap between the ground and excited electronic states is larger than the energy scale of the nuclear motion. The use of ABO to describe lattice motion in metals is, therefore, questionable. Nonetheless, the ABO has proven effective for the determination of chemical reactions, molecular dynamics and phonon frequencies in a wide range of metallic systems.

Quite remarkably ABO fails in graphene [17]. Indeed, the inverse of the G-peak pulsation is $\sim 3 \mathrm{fs}$, which is much smaller than the typical electronmomentum relaxation time, which was estimated to be a few hundred fs 


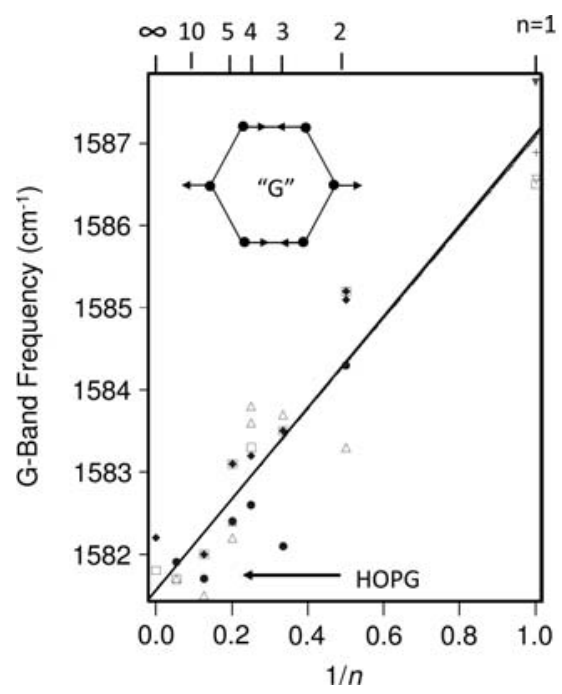

Fig. 16. G-band frequency vs. $1 / n$. Spectra excited with 514.5-nm light and collected from $n$ GLs supported on a single $\mathrm{SiO}_{2}: \mathrm{Si}$ substrate. The straight line is obtained by a least-squares fit. Data on the same set of $n$ GLs were collected from several spots well removed from the edge of the flake

from the electron mobility in graphene [39] and ultrafast spectroscopy in graphite $[114,115]$. Thus, electrons do not have time to relax their momenta to reach the instantaneous adiabatic ground state, as assumed in ABO. The nonadiabatic Born-Oppenheimer calculation for the G-band shift $\Delta \omega$ with $\epsilon_{\mathrm{F}}$ can be described analytically [106]:

$$
\hbar \Delta \omega=\frac{\hbar A\left\langle D_{\Gamma}^{2}\right\rangle_{\mathrm{F}}}{\pi M \omega_{0}\left(\hbar v_{\mathrm{F}}\right)^{2}}\left[\left|\epsilon_{\mathrm{F}}\right|+\frac{\hbar \omega_{0}}{4} \ln \left(\left|\frac{\left|\epsilon_{\mathrm{F}}\right|-\frac{\hbar \omega_{0}}{2}}{\left|\epsilon_{\mathrm{F}}\right|+\frac{\hbar \omega_{0}}{2}}\right|\right)\right]
$$

where $A=5.24 \AA^{2}$ is the graphene unit-cell area and $\omega_{0}$ is the frequency of the $\mathrm{G}$ peak in the undoped case. This equation is derived for $T=0$. However, the result of (12) can be extended to any finite temperature $T[17,106]$, giving reasonable agreement with the experimental data shown in Fig. 15.

A systematic upshift in the position of the $\mathrm{G}$ band with increasing $1 / n$ was reported for $n$ GLs supported on $\mathrm{SiO}_{2}: \mathrm{Si}$ substrates [72, 116, 117]. This behavior is plotted in Fig. 16. Shown there are the results of many spectra collected from $n$ GLs on the same substrate. In most of the cases, a single $n$ GL was measured several times, but with the laser focused on a spot of $\sim 1 \mu \mathrm{m}^{2}$ at different locations on the film. $n$ was determined via atomic force microscope $z$-scans [116]. Although there is a significant amount of scatter in the G-band position for a fixed $n$, a $1 / n$ dependence of the Gband frequency is evident. The solid line in the plot is a least-squares fit to the data, which indicates a $5-\mathrm{cm}^{-1}$ upshift of the $\mathrm{G}$ band from its location 
in highly oriented pyrolytic graphite (HOPG) to that for $n=1$ graphene. Gupta et al. proposed that the upshift may be due to an $n$-dependent bending of the $n \mathrm{GL}$ as it is attracted to the oxide surface and tries to conform to the surface roughness of the substrate, which is about $2 \mathrm{~nm}$. However, as discussed above, this trend might be due to the diminishing influence of self-doping with increasing $n[118,119]$. Indeed, measurements on a variety of single layer graphene have shown a $\mathrm{G}$ peak position variation of $13 \mathrm{~cm}^{-1}$, much bigger than that reported between single and multi-layer graphene $[118,119]$. Another consequence of doping is the significant decrease of $I(2 \mathrm{D}) / I(\mathrm{G})$, with respect to the undoped case [120].

\section{Implications for Phonons and Raman Scattering in Nanotubes}

\subsection{Adiabatic Kohn Anomalies}

Graphene has been used as a model for the calculations of the electronic and vibrational properties of carbon nanotubes. However, a SWNT can be either metallic or semiconducting, whereas graphene is a semimetal/zerogap semiconductor. The differences between graphene and SWNTs can be explained in terms of curvature and confinement [19]. Curvature effects arise because in a nonplanar geometry the $\mathrm{C}-\mathrm{C}$ bonds in SWNTs assume a mixed $\sigma-\pi$ character. Confinement effects arise because the electronic wavefunctions in a SWNT have to be commensurate to the tube circumference, resulting in the quantization of the electronic momentum component perpendicular to the tube axis.

Neglecting the effects of curvature, it is thus possible to map the electronic states of a SWNT onto those of graphene. It has been shown that folding the electronic structure of graphene to describe the band structure of SWNTs produces accurate results for tubes with diameters larger than $0.8 \mathrm{~nm}[121,122]$. This technique is known as electronic zone folding (EZF). In the past, a similar technique had also been applied to phonons. This goes under the name of phonon zone folding (PZF). However, as discussed in Sect. 3.1, phonon dispersions of graphene are affected by two Kohn anomalies, which occur only in metals. Thus, Kohn anomalies cannot be present in semiconducting SWNTs, while they can be enhanced in metallic SWNTs because of their reduced dimensionality [19, 20]. As a consequence, PZF may not be suitable for the description of the phonon dispersion of metallic SWNTs close to the Kohn anomalies. Even for semiconducting SWNTs, PZF of graphene may not be precise, since PZF does not have Kohn anomalies [19]. However, neglecting the effects of curvature, phonons of SWNTs can be obtained from the phonons of a flat graphene sheet, if the calculation is done by performing the electronic Brillouin-zone integration on the lines of the electronic zone 


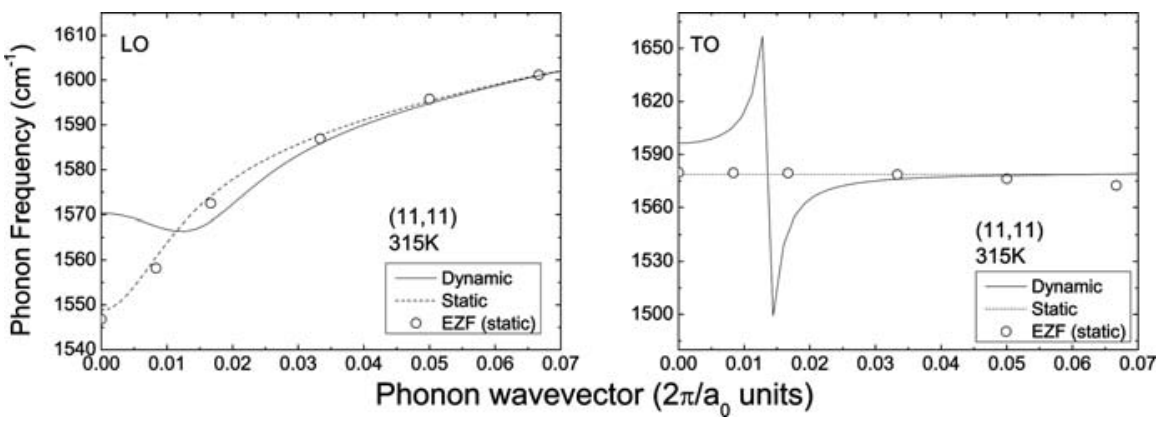

Fig. 17. Phonon dispersion of the modes for a $(11,11)$ SWNT derived from the graphene $E_{2 \mathrm{~g}}$ branch. The dots are the results of static PZF calculations. The dotted and solid lines are obtained with analytical models based, respectively, on the adiabatic and nonadiabatic description of phonons [19]

folding $[19,20]$. This approach allows the description of all the effects of quantum confinement, and can be used to compute phonon dispersions of tubes with a diameter larger than $0.8 \mathrm{~nm}$, which are typical in experiments.

\subsection{Nonadiabatic Kohn Anomalies}

Nonadiabatic effects lead to a deep modification in the description of the Kohn anomalies (KAs) $[17,19,106]$. Using the zone-folding approach of [19, 20], it is possible to obtain the theoretical description of the KAs in metallic SWNTs within the adiabatic (static) and nonadiabatic (dynamic) approaches. These results should then be corrected for curvature effects, as shown by [19]. Figure 17 compares the modes derived from the graphene $E_{2 \mathrm{~g}}$ in a $(11,11)$ metallic SWNT, calculated using a static and a dynamic description of the phonons. In the first case, only the LO mode is affected by a Kohn anomaly, centered at $\boldsymbol{q}=0(\Gamma)$. On the other hand, calculations performed with the dynamic, time-dependent approach show the presence of the anomalies for both the LO and the TO modes, respectively, and predict the anomalies to be shifted from $\boldsymbol{\Gamma}[19]$.

\subsection{The Raman G Peak of Nanotubes}

In SWNTs, the doubly degenerate Raman-active $E_{2 g}$ mode of graphene splits into two dominant phonons. As shown in Fig. 18, such modes are polarized, respectively, along the tube axis (longitudinal mode) and along the tube circumference (tangential mode), and are usually referred to as the LO and the TO modes [19].

In the $1550-1590 \mathrm{~cm}^{-1}$ region, the Raman spectra of SWNTs are characterized by the presence of two distinct features: the so-called $\mathrm{G}^{+}$and $\mathrm{G}^{-}$ 

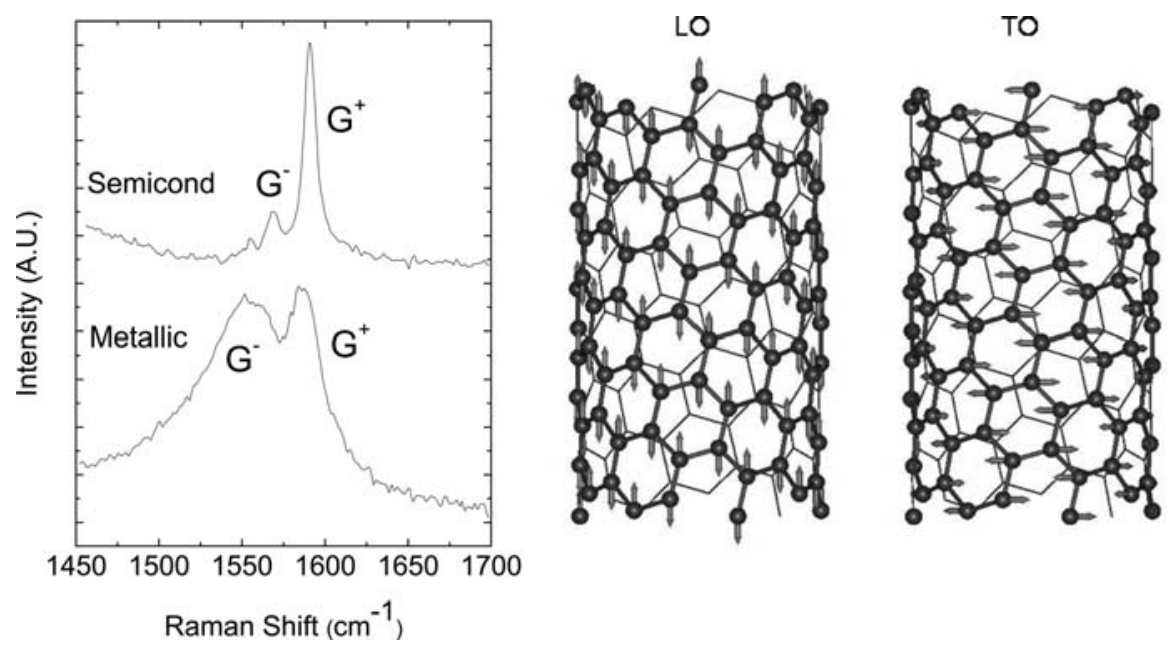

Fig. 18. Left: G-band of semiconducting and metallic SWNTs. Semiconducting tubes are characterized by two sharp peaks. Metallic tubes show a characteristic broad, downshifted $\mathrm{G}^{-}$peak [123]. Right: LO and TO phonon modes of SWNTs derived from the $E_{2 g}$ phonon of graphene

peaks. These peaks are due to the LO and TO modes, and their shape and position strongly depend on the electronic properties of the tubes. The $\mathrm{G}$ band of a metallic and of a semiconducting SWNT [123] are compared in Fig. 18. In semiconducting tubes, both the $\mathrm{G}^{+}$and the $\mathrm{G}^{-}$peaks appear as sharp Lorentzians, centered, respectively, at $\sim 1590 \mathrm{~cm}^{-1}$ and $\sim 1570 \mathrm{~cm}^{-1}$. The $\mathrm{G}^{+}$peak is usually more intense than the $\mathrm{G}^{-}$, and its position is nearly independent of the tube's diameter, whereas the position of the $\mathrm{G}^{-}$peak decreases for decreasing tube diameter. On the other hand, in metallic tubes, the $\mathrm{G}^{-}$ peak is usually rather intense, very broad, and downshifted with respect to its counterpart in semiconducting tubes.

In semiconducting tubes, the splitting between the LO and the TO modes is usually explained in terms of curvature. Indeed, the $\sigma-\pi$ mixing of the $\mathrm{C}-\mathrm{C}$ bonds along the circumference results in a softening of the TO mode with respect to the LO, accounting for both the splitting of the peaks and the diameter dependence of the $\mathrm{G}^{-}$position.

For metallic tubes, different theories have been proposed. The first attempt to explain the broadened, downshifted $\mathrm{G}^{-}$in metallic SWNTs was based on a Fano resonance between the TO phonon and the plasmons in metallic tubes $[124,125]$. However, this model neglects both the presence of Kohn anomalies in the phonon dispersion of metallic SWNTs, and the role of the electron-phonon interaction. Indeed, it is possible to show, as discussed in Sect. 4, that the $\mathrm{G}^{-}$peak of metallic SWNTs is strongly influenced by the effects of EPC [19,20]. Modeling the EPC in metallic SWNTs by using the 


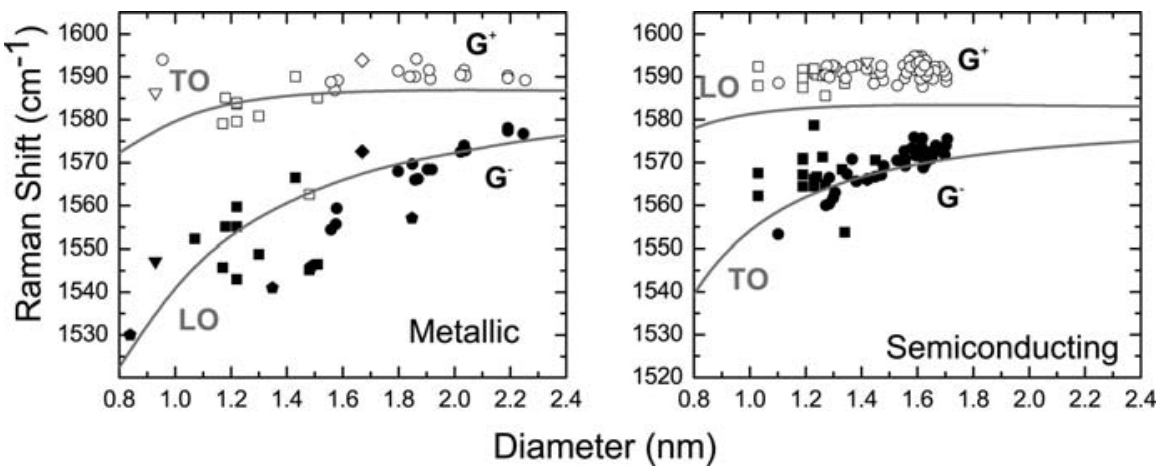

Fig. 19. Comparison between the computed frequency of the LO and the TO phonon in SWNTs (lines) and the experimental position of the $\mathrm{G}^{+}$and the $\mathrm{G}^{-}$Raman peak in metallic and semiconducting tubes as measured by various authors [19]. Calculations include the dynamic effects and a correction for the curvature effects [19]

same tight-binding model as was used for graphene, it is possible to show that the EPC affects the LO mode only and acts in two different ways. First, it is responsible for the onset of a Kohn anomaly resulting in a strong downshift of the LO frequency. Secondly, in agreement with the Fermi golden rule, it reduces the LO-phonon lifetime, resulting in an increase of the phonon linewidth. Consistent with this view, the $\mathrm{G}^{-}$peak in metallic tubes does not originate from a curvature-downshifted TO phonon, as in semiconducting tubes, but is derived from an EPC-affected LO mode [19,20]. The prediction given by the dynamic, time-dependent model can be compared with the data from Raman spectroscopy. Figure 19 shows that the calculated frequencies of the TO and LO modes in metallic SWNTs are in reasonable agreement with the position of the $\mathrm{G}^{+}$and $\mathrm{G}^{-}$Raman peaks. This suggests that the $\mathrm{G}^{+}$ and $\mathrm{G}^{-}$peaks of metallic nanotubes should be assigned to TO (tangential) and LO (axial) modes, the opposite of semiconducting nanotubes [19,20].

In SWNTs, due to the reduced dimensionality, the nonadiabatic contributions are essential to describe the phonons [19] and they should be more important in the case of doped SWNTs [22]. Thus, the shape of the G peak will be affected by doping in a similar fashion to doped graphene. Furthermore, due to energy conservation, measuring a metallic nanotube off resonance can result in a sharp $\mathrm{G}^{-}$feature. We can thus simply understand the reason for the large variety of $\mathrm{G}^{-}$lineshapes reported in the literature. Finally, the nonadiabatic Kohn anomaly at $\Gamma$ also explains the observed electronic temperature dependence of the Raman spectra [19].

It is important to note the following on the shape of the $\mathrm{G}^{-}$peak [20]. The peak shape observed in an actual experiment depends on the coupling between the experimental probe and the system, as discussed in the seminal paper by Fano [126]. Indeed, if the laser couples not only to the $\mathrm{G}^{-}$phonon, 
but also to the continuum that broadens the phonon, the Raman spectrum shows an asymmetric Fano profile [126]. Thus, the detection of an asymmetric Fano profile in a Raman measurement gives information on how the electromagnetic radiation couples to the system, but not on the origin of the broadening. Here, we discussed how the broadening of the $\mathrm{G}^{-}$peak in metallic nanotubes can be mainly attributed to the coupling of the LO phonon to the continuum of the electron-hole excitations, not to phonon-plasmon coupling. This does not imply that a Fano-like profile cannot be observed in experiments.

\section{Outlook}

In the past two years, tens of papers have been published and more than 600 papers have been posted on graphene. Thus, by no means could we give a comprehensive review of this fast-moving field in the limited space available here. So, we have focused on the basic electronic and phonon properties of graphene and their relation to nanotubes.

Even if graphene research is at the beginning, scientists can now exploit the large amount of expertise acquired in the study of carbon nanotubes. Almost all the experiments reported on nanotubes are now being performed on graphene. These range from transistor fabrication, to nanoelectromechanical assemblies, to spin transport to optoelectronics. Furthermore, bilayer and few-layer graphene samples could lead to a variety of interesting new physics and applications. A research area that is still largely unexplored is the growth of graphene layers on a substrate of choice. Large-scale deposition of graphene is the needed breakthrough to make this new material viable for electronic applications. Once this is achieved, standard top-down processes could be utilized for large-area production of devices. This is a major advantage compared to nanotubes, for which on-demand chirality is still a dream.

\section{Acknowledgements}

J. C. Charlier acknowledges the National Fund for Scientific Research [FNRS] of Belgium for financial support. A. C. Ferrari acknowledges support from The Royal Society and The Leverhulme Trust. P. C. Eklund and J. Zhu acknowledge support from the National Science Foundation NIRT program, and we thank A. Gupta and T. J. Russin for helpful discussions and assistance with preparing figures.

\section{References}

[1] P. R. Wallace: The band theory of graphite, Phys. Rev. 71, 622 (1947) 674

[2] L. M. Viculis, J. J. Mack, R. B. Kaner: A chemical route to carbon nanoscrolls, Science 299, 1361 (2003) 674 
[3] L. M. Viculis, J. J. Mack, O. M. Mayer, H. T. Hahn, R. B. Kaner: Intercalation and exfoliation routes to graphite nanoplatelets, J. Mater. Chem. 15, 974 (2005) 674

[4] S. Niyogi, E. Bekyarova, M. E. Itkis, J. L. McWilliams, M. A. Hammon, R. C. Haddon: Solution properties of graphite and graphene, J. Am. Chem. Soc. 128, 7720 (2006) 674

[5] S. Stankovich, D. A. Dikin, G. H. B. Dommett, K. M. Kohlhass, E. J. Zimmey, E. A. Stach, R. D. Piner, S.-B. T. Nguyen, R. S. Ruoff: Graphene-based composite materials, Nature 442, 282 (2006) 674

[6] S. Stankovich, R. D. Piner, X. Chen, N. Wu, T. Nguyen, R. S. Ruoff: Stable aqueous dispersions of graphitic nanoplatelets via the reduction of exfoliated graphite oxide in the presence of poly(sodium 4-styrenesulfonate), J. Mater. Chem. 16, 155 (2006) 674

[7] K. S. Novoselov, A. K. Geim, S. V. Morozov, D. Jiang, S. V. Dubonos, I. V. Girgorieva, A. A. Firsov: Electric field effect in atomically thin carbon films, Science 306, 666 (2004) 674, 686

[8] Y. B. Zhang, J. P. Small, W. V. Pontius, P. Kim: Fabrication and electricfield-dependent transport measurements of mesoscopic graphite devices, Appl. Phys. Lett. 86, 073104 (2005) 674

[9] I. Forbeaux, J. M. Themlin, J. M. Debever: High-temperature graphitization of the 6H-SiC (000(1)over-bar) face, Surf. Sci. 442, 9 (1999) 674

[10] C. Berger, Z. M. Song, T. B. Li, X. B. Li, A. Y. Ogbazghi, R. Feng, Z. T. Dai, A. N. Marchenkov, E. H. Conrad, P. N. First, W. A. de Heer: Ultrathin epitaxial graphite: $2 \mathrm{D}$ electron gas properties and a route toward graphenebased nanoelectronics, J. Phys. Chem. B 108, 19912 (2004) 674

[11] T. Ohta, A. Bostwick, T. Seyller, K. Horn, E. Rotenberg: Controlling the electronic structure of bilayer graphene, Science 313, 951 (2006) 674, 684, 685

[12] E. Rolling, G. H. Gweon, S. Y. Zhou, B. S. Mun, J. L. McChesney, B. S. Hussain, A. Fedorov, P. N. First, W. A. de Heer, A. Lanzara: Synthesis and characterization of atomically thin graphite films on a silicon carbide substrate, J. Phys. Chem. Solids 67, 2172 (2006) 674

[13] K. S. Novoselov, A. K. Geim, S. V. Morozov, D. Jiang, M. I. Katsnelson, I. V. Girgorieva, S. V. Dubonos, A. A. Firsov: Two-dimensional gas of massless Dirac fermions in graphene, Nature 438, 197 (2005) 674, 676, 679, 680, 681

[14] Y. Zhang, Y. W. Tan, H. L. Stormer, P. Kim: Experimental observation of the quantum Hall effect and Berry's phase in graphene, Nature 438, 201 (2005) $674,676,678,679,680,681$

[15] A. K. Geim, K. S. Novoselov: The rise of graphene, Nature Mater. 6, 183 (2007) $674,678,679$

[16] M. Born, R. Oppenheimer: Ann. Phys. 84, 457 (1927) 675, 695

[17] S. Pisana, M. Lazzeri, C. Casiraghi, K. Novoselov, A. K. Geim, A. C. Ferrari, F. Mauri: Breakdown of the adiabatic Born-Oppenheimer approximation in graphene, Nature Mater. 6, 198 (2007) 675, 690, 691, 694, 695, 696, 698

[18] S. Piscanec, M. Lazzeri, F. Mauri, A. Ferrari, J. Robertson: Kohn anomalies and electron-phonon interactions in graphite, Phys. Rev. Lett. 93, 185503 (2004) 675, 688, 689, 691, 693, 694, 695 
[19] S. Piscanec, M. Lazzeri, J. Robertson, A. C. Ferrari, F. Mauri: Optical phonons in carbon nanotubes: Kohn anomalies, Peierls distortions, and dynamic effects, Phys. Rev. B 75, 035427 (2007) 675, 688, 689, 690, 697, 698, 699, 700

[20] M. Lazzeri, S. Piscanec, F. Mauri, A. C. Ferrari, J. Robertson: Phonon linewidths and electron-phonon coupling in graphite and nanotubes, Phys. Rev. B 73, 155426 (2006) 675, 688, 689, 690, 697, 698, 699, 700

[21] M. Lazzeri, S. Piscanec, F. Mauri, A. C. Ferrari, J. Robertson: Electron transport and hot phonons in carbon nanotubes, Phys. Rev. Lett. 95, 236802 (2005) 675, 688, 689

[22] N. Caudal, A. M. Saitta, M. Lazzeri, F. Mauri: Kohn anomalies and nonadiabaticity in doped carbon nanotubes, Phys. Rev. B 75, 115423 (2007) 675, 700

[23] J.-C. Charlier, X. Blase, S. Roche: Electronic and transport properties of nanotubes, Rev. Mod. Phys. 79, 677-732 (2007) 675, 676, 677

[24] J. C. Slonczewski, P. R. Weiss: Band structure of graphite, Phys. Rev. 109, 272 (1958) 678

[25] K. S. Novoselov, D. Jiang, F. Schedin, T. J. Booth, V. V. Khotkevich, S. V. Morozov, A. K. Geim: Two-dimensional atomic crystals, Proc. Nature Acad. Sci. USA 102, 10451 (2005) 678

[26] C. Berger, Z. Song, X. Li, X. Wu, N. Brown, C. Naud, D. Mayou, T. Li, J. Hass, A. N. Marchenkov, E. H. Conrad, P. N. First, W. A. De Heer: Electronic confinement and coherence in patterned epitaxial graphene, Science 312, 1191 (2006) 679

[27] K. Nomura, A. H. MacDonald: Quantum transport of massless Dirac fermions, Phys. Rev. Lett. 98, 076602 (2007) 679

[28] K. S. Novoselov, E. McCann, S. V. Morozov, V. I. F. M. I. Katsnelson, U. Zeitler, D. Jiang, F. Schedin, A. K. Geim: Unconventional quantum Hall effect and Berry's phase of 2 pi in bilayer graphene, Nature Phys. 2, 177 (2006) 679, 685

[29] S. V. Morozov, K. S. Novoselov, M. I. Katsnelson, F. Schedin, L. A. Ponomarenko, D. Jiang, A. K. Geim: Strong suppression of weak localization in graphene, Phys. Rev. Lett. 97, 016801 (2006) 679

[30] V. M. Galitski, S. Adam, S. D. Sarma: Statistics of random voltage fluctuations and the low-density residual conductivity of graphene, arXiv Url: cond-mat/0702117 679

[31] F. D. M. Haldane: Model for a quantum Hall effect without Landau levels: Condensed-matter realization of the "parity anomaly", Phys. Rev. Lett. 61, 2015 (1988) 679

[32] Y. S. Zheng, T. Ando: Hall conductivity of a two-dimensional graphite system, Phys. Rev. B 65, 245420 (2002) 679

[33] M. L. Sadowski, G. Martinez, M. Potemski, C. Berger, W. A. de Heer: Landau level spectroscopy of ultrathin graphite layers, Phys. Rev. Lett. 97, 266405 (2006) 679

[34] Z. Jiang, E. A. Henriksen, L. C. Tung, Y.-J. Wang, M. E. Schwartz, M. Y. Han, P. Kim, H. L. Stormer: Infrared spectroscopy of Landau levels of graphene, Phys. Rev. Lett. 98, 197403 (2007) 679, 680 
[35] R. S. Deacon, K.-C. Chuang, R. J. Nicholas, K. S. Novoselov, A. K. Geim: Cyclotron resonance study of the electron and hole velocity in graphene monolayers, Phys. Rev. B 76 (8), 081406 (2007) 679, 680

[36] K. S. Novoselov, Z. Jiang, Y. Zhang, S. V. Morozov, H. L. Stormer, U. Zeitler, J. C. Maan, G. S. Boebinger, P. Kim, A. K. Geim: Room-temperature quantum Hall effect in graphene, Science 315, 1379 (2007) 679

[37] M. Berry: Quantal phase-factors accompanying adiabatic changes, Proc. R. Soc. Lond. A 392, 45 (1984) 680

[38] D. Shoenberg: Magnetic Oscillations in Metals (Cambridge University Press, Cambridge 1984) 680

[39] Y. Zhang, Z. Jiang, J. P. Small, M. S. Purewal, Y. W. Tan, M. Fazlollahi, J. D. Chudow, J. A. Jaszczak, H. L. Stormer, P. Kim: Landau-level splitting in graphene in high magnetic fields, Phys. Rev. Lett. 96, 136806 (2006) 681, 696

[40] D. A. Abanin, K. S. Novoselov, U. Zeitler, P. A. Lee, A. K. Geim, L. S. Levitov: Dissipative quantum Hall effect in graphene near the Dirac point, Phys. Rev. Lett. 98, 196806 (2007) 681

[41] K. Yang: Spontaneous symmetry breaking and quantum Hall effect in graphene, Solid State Comm. 143, 27 (2007) 681

[42] K. Nakada, M. Fujita, G. Dresselhaus, M. S. Dresselhaus: Edge state in graphene ribbons: Nanometer size effect and edge shape dependence, Phys. Rev. B 54, 17954 (1996) 681, 682

[43] K. Wakabayashi, M. Fujita, H. Ajiki, M. Sigrist: Electronic and magnetic properties of nanographite ribbons, Phys. Rev. B 59, 8271 (1999) 681, 682, 683

[44] Y. Miyamoto, K. Nakada, M. Fujita: First-principles study of edge states of H-terminated graphitic ribbons, Phys. Rev. B 59, 9858 (1999) 681, 682

[45] T. Kawai, Y. Miyamoto, O. Sugino, Y. Koga: Graphitic ribbons without hydrogen-termination: Electronic structures and stabilities, Phys. Rev. B 62, R16349 (2000) 681, 682

[46] S. Okada, A. Oshiyama: Magnetic ordering in hexagonally bonded sheets with first-row elements, Phys. Rev. Lett. 87, 146803 (2001) 681, 682, 683

[47] H. Lee, Y.-W. Sun, N. Park, S. Han, J. Yu: Magnetic ordering at the edges of graphitic fragments: Magnetic tail interactions between the edge-localized states, Phys. Rev. B 72, 174431 (2005) 681, 682, 683

[48] M. Ezawa: Peculiar width dependence of the electronic properties of carbon nanoribbons, Phys. Rev. B 73, 045432 (2006) 681, 682

[49] L. Brey, H. A. Fertig: Electronic states of graphene nanoribbons studied with the Dirac equation, Phys. Rev. B 73, 235411 (2006) 681, 682

[50] K.-I. Sasaki, S. Murakami, R. Saito: J. Phys. Soc. Jpn. 75, 074713 (2006) 681,682

[51] D. A. Abanin, P. A. Lee, L. S. Levitov: Spin-filtered edge states and quantum Hall effect in graphene, Phys. Rev. Lett. 96, 176803 (2006) 681, 682

[52] Y.-W. Son, M. L. Cohen, S. G. Louie: Energy gaps in graphene nanoribbons, Phys. Rev. Lett. 97, 216803 (2006) 681, 682, 683

[53] Y.-W. Son, M. L. Cohen, S. G. Louie: Erratum: Energy gaps in graphene nanoribbons, Phys. Rev. Lett. 98, 089901 (2007) 681, 682, 683

[54] Y.-W. Son, M. L. Cohen, S. G. Louie: Half-metallic graphene nanoribbons, Nature 444, 347 (2006) 681, 682, 683 
[55] Y. Kobayashi, K. Fukui, T. Enoki, K. Kusakabe, Y. Kaburagi: Observation of zigzag and armchair edges of graphite using scanning tunneling microscopy and spectroscopy, Phys. Rev. B 71, 193406 (2005) 682

[56] C. L. Kane, E. J. Mele: Z(2) topological order and the quantum spin Hall effect, Phys. Rev. Lett. 95, 146802 (2005) 683

[57] D. Prezzi, D. Varasano, A. Ruini, E. Molinari: Optical properties of graphene nanoribbons: The role of many-body effects, arXiv:0706.0916 (2007) 683

[58] M. Y. Han, B. Özyilmaz, Y. Zhang, P. Kim: Energy band-gap engineering of graphene nanoribbons, Phys. Rev. Lett. 98, 206805 (2007) 683

[59] Z. Chen, Y.-M. Lin, M. J. Rooks, P. Avouris: Graphene nano-ribbon electronics, arXiv URL: cond-mat/0701599 683

[60] F. Cervantes-Sodi, G. Csanyi, S. Piscanec, A. C. Ferrari: Edge functionalised and substitutional doped graphene nanoribbons: electronic and spin properties, Cond Mat 0711.2340 (2007) 683

[61] G. Dresselhaus, M. S. Dresselhaus: Spin-orbit interaction in graphite, Phys. Rev. 140, 401 (1965) 683

[62] J.-C. Charlier, X. Gonze, J.-P. Michenaud: First-principles study of the electronic properties of graphite, Phys. Rev. B 43, 4579 (1991) 684

[63] J.-C. Charlier, X. Gonze, J.-P. Michenaud: First-principles study of the electronic properties of simple hexagonal graphite, Phys. Rev. B 46, 4531 (1992) 684

[64] J.-C. Charlier, X. Gonze, J.-P. Michenaud: First-principles study of the stacking effect on the electronic properties of graphite(s), Carbon 32, 289-299 (1994) 684

[65] J.-C. Charlier, X. Gonze, J.-P. Michenaud: Graphite interplanar bonding: electronic delocalization and van der Waals interaction, Europhys. Lett. 28, 403-408 (1994) 684

[66] S. Latil, L. Henrard: Charge carriers in few-layer graphene films, Phys. Rev. Lett. 97, 036803 (2006) 684

[67] F. Guinea, A. H. Castro-Neto, N. M. R. Peres: Electronic states and Landau levels in graphene stacks, Phys. Rev. B 73, 245426 (2006) 684

[68] J.-C. Charlier, J.-P. Michenaud, P. Lambin: Tight-binding density of electronic states of pregraphitic carbon, Phys. Rev. B 46, 4540 (1992) 684

[69] E. McCann, V. I. Fal'ko: Landau-level degeneracy and quantum Hall effect in a graphite bilayer, Phys. Rev. Lett. 96, 086805 (2006) 684, 685

[70] E. V. Castro, K. S. Novoselov, S. V. Morozov, N. M. R. Peres, J. M. B. L. dos Santos, J. Nilsson, F. Guinea, A. K. Geim, A. H. Castro-Neto: Biased bilayer graphene: Semiconductor with a gap tunable by electric field effect, arXiv URL: cond-mat/0611342 685

[71] C. Casiraghi, A. Hartschuh, E. Lidorikis, H. Qian, H. Harutyunyan, T. Gokus, K. S. Novoselov, A. C. Ferrari: Rayleigh imaging of graphene and graphene layers, Nano Lett. 7, 2711 (2007) 686

[72] A. C. Ferrari, J. C. Meyer, V. Scardaci, C. Casiraghi, M. Lazzeri, F. Mauri, S. Piscanec, D. Jiang, K. S. Novoselov, S. Roth, A. K. Geim: Raman spectrum of graphene and graphene layers, Phys. Rev. 97, 187401 (2006) 686, 690, 691, 692, 693, 694, 696

[73] A. C. Ferrari, J. Robertson: Raman spectroscopy in carbons: From nanotubes to diamond, Philos. Trans. Roy. Soc. A 362, 2267-2565 (2004) 687 
[74] C. Castiglioni, F. Negri, M. Rigolio, G. Zerbi: Raman activation in disordered graphites of the $\mathrm{A}_{1}^{\prime}$ symmetry forbidden $\mathrm{k} \neq 0$ phonon: The origin of the $\mathrm{D}$ line, J. Chem. Phys. 115, 3769 (2001) 687

[75] C. Castiglioni, M. Tommasini, G. Zerbi: Raman spectroscopy of polyconjugated molecules and materials: Confinement effect in one and two dimensions, Philos. Trans. R. Soc. Lond. A 362, 2425 (2004) 687

[76] F. Tuinstra, J. Koenig.: Raman spectrum of graphite, J. Chem. Phys. 53, 1126 (1970) 687, 688, 693

[77] A. C. Ferrari, J. Robertson: Interpretation of Raman spectra of disordered and amorphous carbon, Phys. Rev. B 61, 14095 (2000) 687, 688, 693

[78] R. J. Nemanich, S. A. Solin: First- and second-order Raman scattering from finite-size crystals of graphite, Phys. Rev. B 20, 392 (1979) 687, 690

[79] R. Al-Jishi, G. Dresselhaus: Lattice-dynamical model for graphite, Phys. Rev. B, 26, $4514(1982) \quad 687$

[80] R. P. Vidano, D. B. Fishbach, L. J. Willis, T. M. Loehr: Observation of Raman band shifting with excitation wavelength for carbons and graphites, Solid State Commun. 39, 341 (1981) 687, 690

[81] I. Pocsik, M. Hundhausen, M. Koos, L. Ley: DC electrical properties of amorphous carbon with different bonding hybridization, J. Non-Cryst. Solids 227230, 1087 (1998) 687, 688

[82] P. Lespade, A. Marchard, M. Couzi, F. Cruege: Caracterisation de materiaux carbones par microspectrometrie Raman, Carbon 22, 375 (1984) 687

[83] C. Thomsen, S. Reich: Double resonant Raman scattering in graphite, Phys. Rev. Lett. 85, 5214 (2000) 687, 688, 691

[84] A. V. Baranov, A. N. Bekhterev, Y. S. Bobovich, V. I. Petrov: Interpretation of some peculiarities in Raman spectra of graphite and glassy carbon, Opt. Spektrosk. 62, 1036 (1987) 687

[85] J. Maultzsch, S. Reich, C. Thomsen, H. Requardt: Phonon dispersion in graphite, P. Ordejón. Phys. Rev. Lett. 92, 075501 (2004) 688

[86] M. J. Matthews, M. A. Pimenta, G. Dresselhaus, M. S. Dresselhaus, M. Endo: Origin of dispersive effects of the Raman D band in carbon materials, Phys. Rev. B 59, 6585 (1999) 687, 688

[87] A. Gruneis, R. Saito, T. Kimura, L. G. Cançado, M. A. Pimenta, A. Jorio, A. G. S. Filho, G. Dresselhaus, M. S. Dresselhaus: Determination of twodimensional phonon dispersion relation of graphite by Raman spectroscopy, Phys. Rev. B 65, 155405 (2002) 687, 688

[88] R. Saito, A. Jorio, A. G. S. Filho, G. Dresselhaus, M. S. Dresselhaus, M. A. Pimenta: Probing phonon dispersion relations of graphite by double resonance Raman scattering, Phys. Rev. Lett. 88, 027401 (2002) 687, 688

[89] A. C. Ferrari, J. Robertson.: Resonant Raman spectroscopy of disordered, amorphous, and diamondlike carbon, Phys. Rev. B, 64, 075414 (2001) 687, 688,691

[90] C. Mapelli, C. Castiglioni, G. Zerbi, K. Mullen: Common force field for graphite and polycyclic aromatic hydrocarbons, Phys. Rev. B 60, 12710 (1999) 688

[91] G. Kresse, J. Furthmuller, J. Hafner: Ab initio force constant approach to phonon dispersion relations of diamond and graphite, Europhys. Lett. 32, 729 (1995) 688 
[92] P. Pavone, R. Bauer, K. Karch, O. Schütt, S. Vent, W. Windl, D. Strauch, S. Baroni, S. de Gironcoli: Ab initio phonon calculations in solids, Physica B, 219-220, 439 (1996) 688

[93] L. Wirtz, A. Rubio: The phonon dispersion of graphite revisited, Solid. State Commun. 131, 141 (2004) 688

[94] O. Dubay, G. Kresse: Accurate density functional calculations for the phonon dispersion relations of graphite layer and carbon nanotubes, Phys. Rev. B 67, $035401(2003) 688$

[95] W. Kohn: Image of the Fermi surface in the vibration spectrum of a metal, Phys. Rev. Lett. 2, 393 (1959) 688

[96] A. Javey, J. Guo, M. Paulsson, Q. Wang, D. Mann, M. Lundstrom, H. Dai: High-field quasiballistic transport in short carbon nanotubes, Phys. Rev. Lett. 92, 106804 (2004) 689

[97] J. Y. Park, S. Rosenblatt, Y. Yaish, V. Sazonova, H. Ustunel, S. Braig, T. A. Arias, P. W. Brouwer, P. L. McEuen: Electron-phonon scattering in metallic single-walled carbon nanotubes, Nano Lett. 4, 517 (2004) 689

[98] V. Perebeinos, J. Tersoff, P. Avouris: Electron-phonon interaction and transport in semiconducting carbon nanotubes, Phys. Rev. Lett. 94, 086802 (2005) 689

[99] Z. Yao, C. L. Kane, C. Dekker: High-field electrical transport in single-wall carbon nanotubes, Phys. Rev. Lett. 84, 2941 (2000) 689

[100] J. Jiang, R. Saito, A. Gruneis, G. Dresselhaus, M. S. Dresselhaus: Electronphonon interaction and relaxation time in graphite, Chem. Phys. Lett. 392, 383 (2004) 689

[101] J. Jiang, R. Saito, A. Grüneis, S. G. Chou, G. G. Samsonidze, A. Jorio, G. Dresselhaus, M. S. Dresselhaus: Photoexcited electron relaxation processes in single-wall carbon nanotubes, Phys. Rev. B 71, 045417 (2005) 689

[102] G. Pennington, N. Goldsman: Semiclassical transport and phonon scattering of electrons in semiconducting carbon nanotubes, Phys. Rev. B 68, 045426 (2004) 689

[103] G. D. Mahan: Electron-optical phonon interaction in carbon nanotubes, Phys. Rev. B 68, 125409 (2003) 689

[104] P. H. Tan, C. Y. Hu, J. Dong, W. C. Shen, B. F. Zhang: Polarization properties, high-order Raman spectra, and frequency asymmetry between Stokes and anti-Stokes scattering of Raman modes in a graphite whisker, Phys. Rev B 64, 214301 (2000) 690

[105] V. Scardaci, P. H. Tan, A. C. Ferrari, et al.: unpublished 690

[106] M. Lazzeri, F. Mauri: Nonadiabatic Kohn anomaly in a doped graphene monolayer, Phys. Rev. Lett. 97, 266407 (2006) 690, 695, 696, 698

[107] A. Gupta, P. C. Eklund: unpublished 691

[108] Y. Wang, D. C. Aolsmeyer, R. L. McCreery: Raman spectroscopy of carbon materials: Structural basis of observed spectra, Chem. Mater. 2, 557 (1990) 691

[109] A. Gupta, P. C. Eklund: unpublished 691

[110] P. H. Tan, C. Casiraghi, A. C. Ferrari: unpublished 693

[111] J. Yan, Y. Zhang, P. Kim, A. Pinczuk: Electric field effect tuning of electronphonon coupling in graphene, Phys. Rev. Lett. 98, 166802 (2007) 694, 695

[112] T. Ando: Anomaly of optical phonon in monolayer graphene, J. Phys. Soc. Jpn. 75, 124701 (2006) 695 
[113] A. H. Castro-Neto, F. Guinea.: Electron-phonon coupling and Raman spectroscopy in graphene, Phys. Rev. B 75, 045404 (2007) 695

[114] G. Moos, C. Gahl, R. Fasel, M. Wolf, T. Hertel: Anisotropy of quasiparticle lifetimes and the role of disorder in graphite from ultrafast time-resolved photoemission spectroscopy, Phys. Rev. Lett. 87, 267402 (2001) 696

[115] T. Kampfrath, L. Perfetti, F. Schapper, C. Frischkorn, M. Wolf: Strongly coupled optical phonons in the ultrafast dynamics of the electronic energy and current relaxation in graphite, Phys. Rev. Lett. 95, 187403 (2005) 696

[116] A. Gupta, G. Chen, P. Joshi, S. Tadigadapa, P. C. Eklund: Raman scattering from high-frequency phonons in supported n-graphene layer films, Nano Lett. 6, 2667 (2006) 696

[117] D. Graf, F. Molitor, K. Ensslin, C. Stampfer, A. Jungen, C. Hierold, L. Wirtz: Spatially resolved Raman spectroscopy of single- and few-layer graphene, Nano Lett. 7, 238 (2007) 696

[118] C. Casiraghi, S. Pisana, K. S. Novoselov, A. K. Geim, A. C. Ferrari: Raman fingerprint of charged impurities in graphene, Cond-Mat 0709.2566 (2007) 697

[119] C. Casiraghi, S. Pisana, K. S. Novoselov, A. K. Geim, A. C. Ferrari: Raman fingerprint of charged impurities in graphene, Appl. Phys. Lett. in press. 697

[120] A. Das, S. Pisana, S. Piscanec, B. Chakraborty, S. K. Saha, U. V. Waghmare, R. Yiang, H. R. Krishnamurhthy, A. K. Geim, A. C. Ferrari, A. K. Sood: Electrochemically gated graphene: Monitoring dopants by Raman scattering, cond mat 0709.1174 (2007) 697

[121] V. Zolyomi, J. Kurti: First-principles calculations for the electronic band structures of small diameter single-wall carbon nanotubes, Phys. Rev. B, 70, 085403 (2004) 697

[122] D. Connétable, D., G.-M. Rignanese, J.-C. Charlier, X. Blase: Room temperature Peierls distortion in small diameter nanotubes, Phys. Rev. Lett. 94, 015503 (2005) 697

[123] A. Jorio, R. Saito, M. S. Dresselhaus, G. Dressselhaus: One contribution of 13 to a theme 'Raman spectroscopy in carbons: from nanotubes to diamond', Trans. Roy. Soc. A 362, 2311 (2004) 699

[124] S. D. M. Brown, A. Jorio, P. Corio, M. S. Dresselhaus, G. Dresselhaus, R. Saito, K. Kneipp: Origin of the Breit-Wigner-Fano lineshape of the tangential g-band feature of metallic carbon nanotubes, Phys. Rev. B 63, 155414 (2001) 699

[125] K. Kempa: Gapless plasmons in carbon nanotubes and their interactions with phonons, Phys. Rev. B 66, 195406 (2002) 699

[126] U. Fano: Effects of configuration interaction on intensities and phase shifts, Phys. Rev. 124, 1866 (1961) 700, 701

\section{Index}

graphene, 676

$\pi$ orbital, 673

$\sigma$-bond, 673
2D peak, 690

band structure, 685

Berry's phase, 676 
bilayer, 685, 693

Born-Oppenheimer approximation, 675,695

$\mathrm{C}-\mathrm{C}$ bond, 673

carrier density, 678

D band, 687

Dirac fermion, 674

Dirac point, 674

doping, 694

double resonance, 687

electron-phonon interaction in graphene, 675

electronic group velocity, 676

epitaxial graphitic film, 674

G band, 687, 696

$\mathrm{G}^{\prime}$ band, 690

graphene FET, 678

half integer quantum Hall effect, 679-681

Hall resistance, 680

integer quantum Hall effect, 674

Kohn anomaly, 675, 686, 697, 698

Landau level, 679

magnetoresistance, 680

massless Dirac fermion, 674 minimum quantum conductivity, 679

mobility, 678

non-Born-Oppenheimer effect, 675

phonon dispersion, 687, 688

Raman linewidth, 689

Raman spectroscopy, 686, 690

resistivity, 678

Shubnikov-de Haas oscillation, 676

single-particle Zeeman splitting, 681

tight-binding Hamiltonian, 676

turbostratic random stacking, 684

nanoribbon, 681

armchair edge, 683

chemically functionalized, 683

many-body correction, 683

self-energy, 683

spin-polarization effect, 683

spintronic, 683

zigzag edge, 683

nanotube, 698

G band, 698

LO mode, 698

TO mode, 698 